\title{
SPARSE POLYNOMIAL APPROXIMATION OF PARAMETRIC ELLIPTIC PDES. PART II: LOGNORMAL COEFFICIENTS*
}

\author{
Markus Bachmayr ${ }^{1}$, Albert Cohen $^{1}$, Ronald DeVore ${ }^{2}$ and Giovanni Migliorati ${ }^{1}$
}

\begin{abstract}
We consider the linear elliptic equation $-\operatorname{div}(a \nabla u)=f$ on some bounded domain $D$, where $a$ has the form $a=\exp (b)$ with $b$ a random function defined as $b(y)=\sum_{j \geq 1} y_{j} \psi_{j}$ where $y=\left(y_{j}\right) \in \mathbb{R}^{\mathbb{N}}$ are i.i.d. standard scalar Gaussian variables and $\left(\psi_{j}\right)_{j \geq 1}$ is a given sequence of functions in $L^{\infty}(D)$. We study the summability properties of Hermite-type expansions of the solution map $y \mapsto u(y) \in V:=H_{0}^{1}(D)$, that is, expansions of the form $u(y)=\sum_{\nu \in \mathcal{F}} u_{\nu} H_{\nu}(y)$, where $H_{\nu}(y)=\prod_{j \geq 1} H_{\nu_{j}}\left(y_{j}\right)$ are the tensorized Hermite polynomials indexed by the set $\mathcal{F}$ of finitely supported sequences of nonnegative integers. Previous results [V.H. Hoang and C. Schwab, M3AS 24 (2014) 797-826] have demonstrated that, for any $0<p \leq 1$, the $\ell^{p}$ summability of the sequence $\left(j\left\|\psi_{j}\right\|_{L^{\infty}}\right)_{j \geq 1}$ implies $\ell^{p}$ summability of the sequence $\left(\left\|u_{\nu}\right\|_{V}\right)_{\nu \in \mathcal{F}}$. Such results ensure convergence rates $n^{-s}$ with $s=\frac{1}{p}-\frac{1}{2}$ of polynomial approximations obtained by best $n$-term truncation of Hermite series, where the error is measured in the mean-square sense, that is, in $L^{2}\left(\mathbb{R}^{\mathbb{N}}, V, \gamma\right)$, where $\gamma$ is the infinite-dimensional Gaussian measure. In this paper we considerably improve these results by providing sufficient conditions for the $\ell^{p}$ summability of $\left(\left\|u_{\nu}\right\|_{V}\right)_{\nu \in \mathcal{F}}$ expressed in terms of the pointwise summability properties of the sequence $\left(\left|\psi_{j}\right|\right)_{j \geq 1}$. This leads to a refined analysis which takes into account the amount of overlap between the supports of the $\psi_{j}$. For instance, in the case of disjoint supports, our results imply that, for all $0<p<2$ the $\ell^{p}$ summability of $\left(\left\|u_{\nu}\right\|_{V}\right)_{\nu \in \mathcal{F}}$ follows from the weaker assumption that $\left(\left\|\psi_{j}\right\|_{L^{\infty}}\right)_{j \geq 1}$ is $\ell^{q}$ summable for $q:=\frac{2 p}{2-p}>p$. In the case of arbitrary supports, our results imply that the $\ell^{p}$ summability of $\left(\left\|u_{\nu}\right\|_{V}\right)_{\nu \in \mathcal{F}}$ follows from the $\ell^{p}$ summability of $\left(j^{\beta}\left\|\psi_{j}\right\|_{L^{\infty}}\right)_{j \geq 1}$ for some $\beta>\frac{1}{2}$, which still represents an improvement over the condition in [V.H. Hoang and C. Schwab, M3AS 24 (2014) 797-826]. We also explore intermediate cases of functions with local yet overlapping supports, such as wavelet bases. One interesting observation following from our analysis is that for certain relevant examples, the use of the Karhunen-Loève basis for the representation of $b$ might be suboptimal compared to other representations, in terms of the resulting summability properties of $\left(\left\|u_{\nu}\right\|_{V}\right)_{\nu \in \mathcal{F}}$. While we focus on the diffusion equation, our analysis applies to other type of linear PDEs with similar lognormal dependence in the coefficients.
\end{abstract}

Mathematics Subject Classification. 41A10, 41A58, 41A63, 65N15, 65T60.

Received September 27, 2015. Revised June 9, 2016. Accepted July 16, 2016.

\footnotetext{
Keywords and phrases. Stochastic PDEs, lognormal coefficients, $n$-term approximation, Hermite polynomials.

* This research was supported by ONR Contracts N00014-15-1-2181 and N0014-16-2706; the NSF Grant DMS 1521067; the DARPA Grant HR0011619523 through Oak Ridge National Laboratory; the Institut Universitaire de France; and the ERC AdG 338977 BREAD.

1 Sorbonne Universités, UPMC Univ Paris 06, CNRS, UMR 7598, Laboratoire Jacques-Louis Lions, 4 place Jussieu, 75005 Paris, France. bachmayr@ljll.math.upmc.fr; cohen@ljll.math.upmc.fr; migliorati@ljll.math.upmc.fr

2 Department of Mathematics, Texas A\&M University, College Station, TX 77840, USA. rdevore@math.tamu.edu
} 


\section{INTRODUCTION}

\subsection{Approximation of high-dimensional parametric PDEs}

Parametric partial differential equations have the general form

$$
\mathcal{D}(u, y)=0,
$$

where $u \mapsto \mathcal{D}(u, y)$ is a partial differential operator that depends on a parameter vector $y=\left(y_{j}\right)_{j=1, \ldots, J}$ ranging in a certain domain $U \in \mathbb{R}^{J}$, where $J \geq 1$ is fixed. Assuming well-posedness of the problem in some Banach space $V$, the solution map

$$
y \mapsto u(y),
$$

is defined from the parameter domain $U$ to the solution space $V$.

Equations of this type arise both in stochastic and deterministic modeling, depending on the nature of the parameters $y_{j}$ which may either be random or deterministic variables. In both settings, one main computational challenge is to approximate the entire solution map $y \mapsto u(y)$ up to a prescribed accuracy, with reasonable computational cost. This task has been intensively studied since the $1990 \mathrm{~s}$, see in particular $[14,15,20,23]$ for general treatments. It becomes very challenging when the number of parameters $J$ is large due to the curse of dimensionality. Ideally, one would like to design numerical methods that are immune to the growth of $J$, which in principle amounts to treat the case of countably many variables, that is,

$$
y=\left(y_{j}\right)_{j \geq 1} \in U \subset \mathbb{R}^{\mathbb{N}} .
$$

This problem has been the object of much attention in recent years $[3,4,6,8,9,13,16,19]$.

Sparse polynomial methods are based on approximations to $u$ of the form

$$
u_{\Lambda}(y):=\sum_{\nu \in \Lambda} u_{\nu} y^{\nu}
$$

where $\Lambda \subset \mathcal{F}$ is a finite set of (multi-)indices $\nu=\left(\nu_{j}\right)_{j \geq 1} \in \mathcal{F}$ and $y^{\nu}=\prod_{j \geq 1} y_{j}^{\nu_{j}}$. In the case of an infinite number of parameters, the index set $\mathcal{F}$ denotes the (countable) set of all sequences of nonnegative integers which are finitely supported (i.e. those sequences for which only finitely many terms are nonzero). Note that the polynomial coefficients $u_{\nu}$ are functions in $V$, and therefore the construction of $u_{\Lambda}$ requires in principle the computation of \#( $\Lambda)$ such functions.

One particularly relevant example is the model elliptic PDE

$$
-\operatorname{div}(a \nabla u)=f,
$$

set on a bounded Lipschitz domain $D \subset \mathbb{R}^{d}$ with homogeneous Dirichlet boundary conditions (in typical applications $d=1,2,3)$, where $a=a(y)$ is a diffusion coefficient that depends on $y$, and where $f \in H^{-1}(D)$ is fixed. The so-called affine case refers to a diffusion coefficient of the form

$$
a(y)=\bar{a}+\sum_{j \geq 1} y_{j} \psi_{j}
$$

where $\bar{a}$ and the $\left(\psi_{j}\right)_{j \geq 1}$ are given functions from $L^{\infty}(D)$. In this case, the $y_{j}$ typically range on finite intervals which upon renormalization of $\bar{a}$ and $\psi_{j}$ can be assumed to be $[-1,1]$. Therefore the parameter domain is the infinite-dimensional box

$$
U:=[-1,1]^{\mathbb{N}},
$$

and well-posedness of the problem in $V=H_{0}^{1}(D)$ is ensured for all $y \in U$ by the so-called uniform ellipticity assumption

$$
\sum_{j \geq 1}\left|\psi_{j}(x)\right| \leq \bar{a}(x)-r, \quad x \in D,
$$


for some fixed $r>0$. For this model problem, convergence results have been obtained for polynomial approximations $u_{\Lambda_{n}}$, where $\#\left(\Lambda_{n}\right)=n$, constructed by best $n$-term truncation of infinite polynomial expansions, either of Taylor or Legendre type, that is, by retaining the $n$ coefficients of largest norms in such expansions.

A striking result, first established in [9] under the uniform ellipticity assumption, states that whenever the sequence $\left(\left\|\psi_{j}\right\|_{L^{\infty}}\right)_{j \geq 1}$ is $\ell^{p}$ summable for some $0<p<1$, then such $n$-term polynomial approximations converge with rate $n^{-s}$ in $L^{\infty}(U, V)$ where $s:=\frac{1}{p}-1$. For the Legendre approximations, an improved rate $n^{-s}$, with $s:=\frac{1}{p}-\frac{1}{2}$, is achieved in $L^{2}(U, V, \mu)$ where $\mu$ is the multivariate uniform measure, that is, in the mean-square sense if the $y_{j}$ are i.i.d. uniformly distributed variables. These results have been extended to a large range of linear or nonlinear parametric PDEs $[6,8]$ where $y$ is again ranging in the infinite-dimensional box $U$. They heavily rely on the holomorphy of the solution map $y \mapsto u(y)$ in each variable $y_{j}$.

\subsection{Elliptic PDEs with lognormal coefficients}

In the present paper, we focus our attention on the so-called lognormal case for the elliptic PDE (1.5). In this case, the diffusion coeffient $a$ is of the form

$$
a=\exp (b)
$$

where $b$ is a random function of the form

$$
b=b(y)=\sum_{j \geq 1} y_{j} \psi_{j}
$$

defined from a given sequence $\left(\psi_{j}\right)_{j \geq 1}$ of functions in $L^{\infty}(D)$, with $y=\left(y_{j}\right)_{j \geq 1}$ a sequence of i.i.d. $\mathcal{N}(0,1)$ random variables. Thus, the parameter vector $y$ now ranges over the unbounded domain

$$
U:=\mathbb{R}^{\mathbb{N}} .
$$

We work with the usual product measure space given by

$$
(U, \mathcal{B}(U), \gamma)=\left(\mathbb{R}^{\mathbb{N}}, \mathcal{B}\left(\mathbb{R}^{\mathbb{N}}\right), \gamma\right)
$$

where $\mathcal{B}(U)=\mathcal{B}\left(\mathbb{R}^{\mathbb{N}}\right)$ denotes the $\Sigma$-algebra generated by the Borel cylinders and $\gamma$ the tensorized Gaussian probability measure.

We discuss further several conditions on the family $\left(\psi_{j}\right)_{j \geq 1}$ which ensure that the series in (1.10) converges almost surely in $L^{\infty}(D)$. Under such conditions, $b(y)$ is a Gaussian random variable with values in $L^{\infty}(D)$. By the Lax-Milgram lemma, the associated weak solution $u(y)$ of $(1.5)$ with $a=a(y)=\exp (b(y))$ is a random variable with values in $V=H_{0}^{1}(D)$.

One frequently used approach that leads to this framework is by starting with $b=(b(x))_{x \in D}$ defined as a centered Gaussian process over the domain $D$, with prescribed covariance function

$$
C_{b}\left(x, x^{\prime}\right):=\mathbb{E}\left(b(x) b\left(x^{\prime}\right)\right), \quad x, x^{\prime} \in D .
$$

One then obtains a representation of the form (1.10) by considering the Karhunen-Loève decomposition

$$
b=b(\xi)=\sum_{j \geq 1} \xi_{j} \varphi_{j}
$$

Here $\left(\varphi_{j}\right)_{j \geq 1}$ are the $L^{2}$-orthonormal basis of eigenfunctions of the integral operator with kernel $C_{b}$ and $\xi_{j}$ are independent centered Gaussian variables. One then sets

$$
y_{j}:=\lambda_{j}^{-1 / 2} \xi_{j} \quad \text { and } \quad \psi_{j}:=\lambda_{j}^{1 / 2} \varphi_{j}, \quad \lambda_{j}:=\mathbb{E}\left(\xi_{j}^{2}\right) .
$$


Here again, conditions on $\left(\psi_{j}\right)_{j \geq 1}$ are needed in order to ensure that the series in (1.10) converges almost surely in $L^{\infty}(D)$.

In this paper, we prefer to start with $b$ defined through (1.10) for a more general family $\left(\psi_{j}\right)_{j \geq 1}$. In particular we want to consider cases where the family $\left(\psi_{j}\right)_{j \geq 1}$ in (1.10) is nonorthogonal and therefore differs from a normalized Karhunen-Loève basis of $b$. One typical example is the case where $b$ is a one-dimensional Brownian motion, which has a natural expansion in terms of the nonorthogonal Schauder basis. As shown later in this paper, this representation appears to be more efficient than the Karhunen-Loève expansion in terms of the resulting best $n$-term polynomial approximation rates.

In contrast to the affine model, the lognormal model is intrinsically stochastic: it reflects the situation where the diffusion coefficient $a$ is allowed to become arbitrarily small or large (unlike in the affine case with the uniform ellipticity assumption), however with the probability being controlled by the Gaussian distribution. In this case, the relevant polynomial expansion is the orthonormal Hermite series,

$$
u=\sum_{\nu \in \mathcal{F}} u_{\nu} H_{\nu}, \quad u_{\nu}:=\int_{U} u(y) H_{\nu}(y) \mathrm{d} \gamma(y), \quad H_{\nu}(y)=\prod_{j \geq 1} H_{\nu_{j}}\left(y_{j}\right),
$$

where the univariate Hermite polynomials $H_{k}$ are normalized in $L^{2}(\mathbb{R}, d g)$, with $g$ denoting the standard univariate Gaussian density. Note that $\left(H_{\nu}\right)_{\nu \in \mathcal{F}}$ is an orthonormal basis for the Hilbert space $L^{2}(U, \mathbb{R}, \gamma)$. The coefficients $u_{\nu}$ are well defined in $V$ and the above series converges in the Bochner space $L^{2}(U, V, \gamma)$, which can be identified with $L^{2}(U, \mathbb{R}, \gamma) \otimes V$, whenever $u$ belongs to $L^{2}(U, V, \gamma)$.

Note that a function $u: y \mapsto u(y)$ in $L^{2}(U, V, \gamma)$ may also formally be viewed as a function $(x, y) \mapsto u(x, y):=$ $u(y)(x)$ of both variables $x \in D$ and $y \in U$, and the inner product between two functions $u, v \in L^{2}(U, V, \gamma)$ has the expression

$$
\langle u, v\rangle=\int_{U} \int_{D} \nabla u(x, y) \cdot \nabla v(x, y) \mathrm{d} x \mathrm{~d} \gamma(y)
$$

where the operator $\nabla$ is always meant in the $x$ variable. In order to simplify notation, we shall suppress the $x$-dependence and write the above integral as

$$
\langle u, v\rangle=\int_{U} \int_{D} \nabla u(y) \cdot \nabla v(y) \mathrm{d} x \mathrm{~d} \gamma(y) .
$$

The lognormal model and its approximation have been studied in various papers, e.g. $[1,5,10,12,13,16,17$, $19,21,22]$. Sufficient conditions for the finiteness of moments $\mathbb{E}\left(\|u(y)\|_{V}^{k}\right)$ at all orders $0 \leq k<\infty$ have been established, either by assuming smoothness properties of the covariance kernel $C_{b}$ or by assuming a summability property for the sequence $\left(\left\|\psi_{j}\right\|_{L^{\infty}(D)}\right)_{j \geq 1}$, see for example $[5,10,19]$. Since

$$
\mathbb{E}\left(\|u(y)\|_{V}^{2}\right)=\|u\|_{L^{2}(U, V, \gamma)}^{2}=\sum_{\nu \in \mathcal{F}}\left\|u_{\nu}\right\|_{V}^{2}
$$

the finiteness of the second moment is a necessary and sufficient condition for the $\ell^{2}$ summability of the sequence $\left(\left\|u_{\nu}\right\|_{V}\right)_{\nu \in \mathcal{F}}$ and the convergence of the Hermite series in $L^{2}(U, V, \gamma)$. However, in order to prove convergence rates for the best $n$-term truncation

$$
u_{\Lambda_{n}}:=\sum_{\nu \in \Lambda_{n}} u_{\nu} H_{\nu}
$$

of (1.16), where $\Lambda_{n}$ denotes the set of indices corresponding to the $n$ largest $\left\|u_{\nu}\right\|_{V}$, we need to study the $\ell^{p}$ summability properties of $\left(\left\|u_{\nu}\right\|_{V}\right)_{\nu \in \mathcal{F}}$ for $p<2$. For example, if this sequence is proven to be $\ell^{p}$ summable, then a direct application of standard nonlinear approximation results in sequence spaces, originally due to Stechkin $[8,9,11]$, gives

$$
\left\|u-u_{\Lambda_{n}}\right\|_{L^{2}(U, V, \gamma)}=\left(\sum_{\nu \notin \Lambda_{n}}\left\|u_{\nu}\right\|_{V}^{2}\right)^{1 / 2} \leq C(n+1)^{-s},
$$

where $s:=\frac{1}{p}-\frac{1}{2}$ and $C:=\left\|\left(\left\|u_{\nu}\right\|_{V}\right)_{\nu \in \mathcal{F}}\right\|_{\ell^{p}(\mathcal{F})}$. 
The first and currently the only available result concerning $\ell^{p}$ summability of Hermite coefficients, in the infinite dimensional framework, has been established in [19] and reads as follows.

Theorem 1.1. For any $0<p \leq 1$, if $\left(j\left\|\psi_{j}\right\|_{L^{\infty}}\right)_{j \geq 1} \in \ell^{p}(\mathbb{N})$ then $\left(\left\|u_{\nu}\right\|_{V}\right)_{\nu \in \mathcal{F}} \in \ell^{p}(\mathcal{F})$.

The Hermite coefficients studied in [19] are actually different from those defined in (1.16), since the authors are interested in a specific approximation process, namely the Galerkin method. One specific difficulty in this approach is the fact that the bilinear form

$$
B(u, v)=\int_{U} \int_{D} a(y) \nabla u(y) \cdot \nabla v(y) \mathrm{d} x \mathrm{~d} \gamma(y),
$$

is not continuous and coercive on $L^{2}(U, V, \gamma)$. Thus, when applying the Galerkin method in the space

$$
V_{\Lambda_{n}}:=\left\{\sum_{\nu \in \Lambda_{n}} w_{\nu} H_{\nu}: w_{\nu} \in V\right\},
$$

using a weak formulation based on this bilinear form, one cannot apply Cea's lemma to control the Galerkin error by the projection error $\left\|u-u_{\Lambda_{n}}\right\|_{L^{2}(U, V, \gamma)}=\left(\sum_{\nu \notin \Lambda_{n}}\left\|u_{\nu}\right\|_{V}^{2}\right)^{1 / 2}$. This particular issue has been dealt with in papers such as $[5,13,16,22]$. The approach from [16], which is also used in [19], is based on applying the Galerkin method based on a modified bilinear form where $\gamma$ is replaced by an auxiliary Gaussian measure. The Galerkin error can then be estimated by the $\ell^{2}$ tails of modified Hermite coefficients corresponding to a further auxiliary Gaussian measure. However, inspection of the proof of Theorem 1.1 in [19] shows that it also holds for the standard Hermite coefficients. Note that this issue is specific to the Galerkin method, and does not occur in pseudo-spectral or least-squares methods.

In the present paper, we only consider the classical Hermite coefficients in (1.16), since our main interest is to obtain general polynomial approximation results for the solution map, that may further serve as a benchmark for any numerical method based on such approximations.

The above Theorem 1.1 is comparable to those obtained in [9] for Taylor and Legendre coefficients in the affine case, except for the appearance of the additional factor $j$ in front of $\left\|\psi_{j}\right\|_{L^{\infty}}$, which makes the summability assumption more restrictive. On the one hand, it is quite remarkable that such a result exists, since the complex variable arguments used in the affine case, based solely on holomorphy of the solution map, are not sufficient to control the Hermite coefficients. The proof of the above result in [19] relies, instead, on estimates for the mixed partial derivatives of $u$ up to some finite order $m$ related to $p$. On the other hand, the type of assumptions used in this result indicates that it is not in the sharpest possible form as we now explain.

First, the above result does not cover $\ell^{p}$ summability for $1<p<2$, which one might expect to be obtainable under weaker assumptions. Second, and more importantly, the summability conditions imposed on the $\left\|\psi_{j}\right\|_{L^{\infty}}$ in these results become quite strong and artificial in the case when the supports of these functions do not overlap too much.

As a relevant example for this second point, consider the case where the $\left(\psi_{j}\right)_{j \geq 1}$ are a wavelet basis on the domain $D$. In this case it is more natural to index such bases according to $\left(\psi_{\lambda}\right)_{\lambda \in \nabla}$, where $\lambda$ is a scale-space index following the usual terminology, such as in [7]. With the notation $l=|\lambda|$ for the scale level, there are $\mathcal{O}\left(2^{d l}\right)$ wavelets at this level and each of them has support of diameter $\mathcal{O}\left(2^{-l}\right)$. The supports of wavelets at a given scale $l$ have finite overlap, in the sense that any $x \in D$ is contained in the support of at most $M$ wavelets of level $l$ where $M$ is independent of $x$ and $l$. We assume that the $L^{\infty}$ norms of the wavelets only depend on the scale level, that is,

$$
\left\|\psi_{\lambda}\right\|_{L^{\infty}}=c_{l}, \quad|\lambda|=l .
$$

It is well known that the geometric rate of decay of the wavelet contributions, as the scale level grows, reflects the amount of smoothness in the expansion (or the smoothness of the correlation function in the case of a random series). It is thus natural to study the situation where $c_{l}$ is of the form

$$
c_{l}:=C 2^{-\alpha l},
$$


for some given $\alpha>0$. Then, it can be checked, for example using the arguments from [5], which are recalled further in Section 2, that for arbitrary $\alpha>0$ and $C>0$, the bound (1.25) implies that the solution map has bounded moments $\mathbb{E}\left(\|u(y)\|_{V}^{k}\right)$ for all $0 \leq k<\infty$. Indeed, this follows from the Lax-Milgram lemma and the fact that $\mathbb{E}\left(\exp \left(k\|b(y)\|_{L^{\infty}}\right)\right)$ is finite. The particular case $k=2$ implies that the Hermite coefficient sequence $\left(\left\|u_{\nu}\right\|_{V}\right)_{\nu \in \mathcal{F}}$ belongs to $\ell^{2}(\mathcal{F})$, provided only that $\alpha>0$. However, if we want to use the above mentioned result from [19] to prove $\ell^{p}$ summability of this sequence for values of $p<2$, the appearance of the factor $j$, suddenly, requires the very strong constraint $\alpha>2 d$ which implies a strong smoothness condition on the diffusion coefficients.

The above wavelet example reveals a gap in the currently available analysis: $\ell^{2}$ summability can be obtained under mild assumptions on the smoothness of the diffusion coefficient, while proving $\ell^{p}$ summability for $p<2$ by the existing results immediatley imposes much higher smoothness (in the sense of the required decay of $\left\|\psi_{j}\right\|_{L^{\infty}}$ as $\left.j \rightarrow \infty\right)$. The goal of the present paper is to propose a sharper analysis, which removes this gap. Let us mention that the same gap occurs, in a slightly less pronounced form, for the Legendre coefficients in the affine case, an issue which we address in [2], the first part of the present work.

\subsection{Main results and outline of the paper}

The main result of our paper for the model elliptic equation (1.5) with lognormal diffusion coefficient is the following.

Theorem 1.2. Let $p<2$ and let $q=q(p):=\frac{2 p}{2-p}$. Assume that there exists a positive sequence $\left(\rho_{j}\right)_{j \geq 1}$ such that

$$
\sup _{x \in D} \sum_{j \geq 1} \rho_{j}\left|\psi_{j}(x)\right|<\infty .
$$

and

$$
\left(\rho_{j}^{-1}\right)_{j \geq 1} \in \ell^{q}(\mathbb{N}) .
$$

Then, the solution map $y \mapsto u(y)$ belongs to $L^{k}(U, V, \gamma)$ for all $0 \leq k<\infty$. Moreover, $\left(\left\|u_{\nu}\right\|_{V}\right)_{\nu \in \mathcal{F}} \in \ell^{p}(\mathcal{F})$. In particular, best $n$-term Hermite approximations converge in $L^{2}(U, V, \gamma)$ with rate $n^{-s}$ where $s=\frac{1}{p}-\frac{1}{2}=\frac{1}{q}$.

This theorem gives a significant improvement over Theorem 1.1 in the case where the functions $\psi_{j}$ do not overlap too much. First of all, in the case of disjoint supports, the first condition in Theorem 1.2 is met when $\rho_{j}^{-1}:=\left\|\psi_{j}\right\|_{L^{\infty}}$. Therefore, it implies that for all $0<p<2$ the $\ell^{p}$ summability of $\left(\left\|u_{\nu}\right\|_{V}\right)_{\nu \in \mathcal{F}}$ follows from the assumption that $\left(\left\|\psi_{j}\right\|_{L^{\infty}}\right)_{j \geq 1}$ is $\ell^{q}$ summable for $q=q(p):=\frac{2 p}{2-p}$. Note that $q(p)>p$ for any $p>0$ and that

$$
\lim _{p \rightarrow 2} q(p)=+\infty
$$

which shows that almost no decay of $\left(\left\|\psi_{j}\right\|_{L^{\infty}}\right)_{j \geq 1}$ is required as $p$ gets closer to 2. Secondly, as is shown later in this paper, we can also use Theorem 1.2 to treat the above mentioned wavelet case, and obtain $\ell^{p}$ summability results with $p<2$ for any smoothness index $\alpha>0$. Finally, even in the case of arbitrarily supported $\psi_{j}$, we establish as a corollary of Theorem 1.2 that the $\ell^{p}$ summability of $\left(\left\|u_{\nu}\right\|_{V}\right)_{\nu \in \mathcal{F}}$ follows from the $\ell^{q}$ summability of $\left(\left\|\psi_{j}\right\|_{L^{\infty}}\right)_{j \geq 1}$ with $q:=\frac{2 p}{2-p}$, which again represents a significant improvement over the condition in Theorem 1.1, since this $\ell^{q}$ summability follows by Hölder's inequality from the $\ell^{p}$ summability of $\left(j^{\beta}\left\|\psi_{j}\right\|_{L^{\infty}}\right)_{j \geq 1}$ for some $\beta>\frac{1}{2}$.

Let us mention that in the first part [2] of the present work, a result analogous to Theorem 1.2 is established for Taylor and Legendre coefficients in the affine case, under the additional uniform ellipticity assumption. A common feature with the present paper is that one key ingredient of both proofs consists in establishing certain weighted $\ell^{2}$ estimates for the coefficients $\left\|u_{\nu}\right\|_{V}$ which further translate into $\ell^{p}$ estimates by Hölder's inequality. However, establishing such weighted $\ell^{2}$ estimates for the Hermite coefficients in the present lognormal cases uses completely different techniques than those in [2]. In particular, similar to [19], we only rely on the study 
of mixed partial derivatives $\partial^{\mu} u$ for a limited order $\|\mu\|_{\ell^{\infty}} \leq r$, while the estimates in [2] exploit all orders $\mu \in \mathcal{F}$. Let us also mention that, while we focus on the diffusion equation, inspection of our proofs reveals that our main results can be extended to other types of linear elliptic or parabolic PDEs with similar lognormal dependence of the coefficients. The types of PDEs that can be covered in this way are similar to those that can be treated in the affine case, see the discussion at the end of Section 3 in [2].

The rest of our paper is organized as follows. We begin in Section 2 by revisiting conditions which ensure that $y \mapsto u(y)$ is a measurable map with values in $V$ and with finite moments $\mathbb{E}\left(\|u(y)\|_{V}^{k}\right)$ for all $0 \leq k<\infty$. We introduce a sufficient assumption in terms of the convergence in $L^{\infty}(D)$ of the series $\sum_{j \geq 1} \rho_{j}\left|\psi_{j}\right|$ for a positive sequence $\left(\rho_{j}\right)_{j \geq 1}$ which satisfies the summability property

$$
\sum_{j \geq 1} \exp \left(-\rho_{j}^{2}\right)<\infty
$$

This is a very weak condition on the sequence $\left(\rho_{j}\right)_{j \geq 1}$. In particular, this condition always holds under the assumptions in Theorem 1.2.

As a first step in the proof of Theorem 1.2, we relate in Section 3 the norms $\left\|u_{\nu}\right\|_{V}$ of Hermite coefficients with $\left\|\partial^{\mu} u\right\|_{L^{2}(U, V, \gamma)}$ for relevant values of $\mu$. This leads us to an identity between certain weighted $\ell^{2}$ norms of both quantities. As mentioned above, while all $\nu \in \mathcal{F}$ are considered for the Hermite coefficients, only limited order $\|\mu\|_{\ell^{\infty}} \leq r$ are considered for the partial derivatives. As a second step, we obtain in Section 4 bounds on the previously introduced weighted $\ell^{2}$ norms of the $\left\|\partial^{\mu} u\right\|_{L^{2}(U, V, \gamma)}$ under the assumption that $\sum_{j \geq 1} \rho_{j}\left|\psi_{j}(x)\right|$ is bounded by a relevant constant. Finally, we combine these ingredients in Section 5 to complete the proof of Theorem 1.2.

In Section 6, we derive various consequences of Theorem 1.2 corresponding to the different cases outlined above for the functions $\left(\psi_{j}\right)_{j \geq 1}$ : (i) disjoint or finitely overlapping supports; (ii) wavelets; (iii) arbitrary supports. We discuss, in all three cases, the improvements over Theorem 1.1.

We conclude in Section 7 with an interesting observation which follows from our analysis in the particular case where $b$ is a Brownian motion. For this case we compare the convergence of the best $n$-term truncation of Hermite series when using either the Karhunen-Loève representation or the Schauder basis representation for $b$. The first representation satisfies by construction an $L^{2}$ orthogonality between the $\psi_{j}$, while the second one does not. Due to the limited amount of smoothness of $b$, the assumptions of Theorem 1.2 do not hold for the Karhunen-Loève representation, so that no algebraic convergence rate can be established for the resulting polynomial approximation with our currently available techniques. In contrast, we can obtain an algebraic convergence rate when using the Schauder representation, by exploiting the local support properties of the basis functions. This hints that, in relevant practical cases, the Karhunen-Loève representation might be suboptimal in terms of the resulting polynomial approximation rates for the solution map $y \mapsto u(y)$ in $L^{2}(U, V, \gamma)$.

Let us stress that our results only quantify the approximability of the solution map. They should therefore be viewed as benchmark for concrete numerical methods. The development of numerical methods that provably meet such benchmarks will be the object of further investigation.

\section{MeAsurability AND INTEGRABility of THE SOlUtion MAP}

The first question we investigate is what conditions on $\left(\psi_{j}\right)_{j \geq 1}$ guarantee that the solution map $y \mapsto u(y)$ is in $L^{k}(U, V, \gamma)$, i.e. it is measurable with values in $V$ and satisfies

$$
\mathbb{E}\left(\|u(y)\|_{V}^{k}\right)=\int_{U}\|u(y)\|_{V}^{k} \mathrm{~d} \gamma(y)<\infty
$$

We recall that a function from a measurable space to a Banach space $B$ is measurable (also sometimes called $\mu$-measurable, or Bochner-measurable, or strongly measurable), if and only if it is the almost everywhere pointwise limit of a sequence of simple functions. 
Recall that $u(y)$ is the weak solution to the diffusion equation

$$
-\operatorname{div}(a(y) \nabla u(y))=f,
$$

on the bounded domain $D$ with homogeneous Dirichlet boundary condition, for a fixed $f \in H^{-1}(D)$, where

$$
a(y):=\exp (b(y)) .
$$

The solution $u(y)$ is well defined as an element of $V=H_{0}^{1}(D)$ provided that $\sum_{j \geq 1} y_{j} \psi_{j}$ defines a function $b(y) \in L^{\infty}(D)$. In such a case, by the Lax-Milgram lemma, we have

$$
\|u(y)\|_{V} \leq C\left\|a(y)^{-1}\right\|_{L^{\infty}} \leq C \exp \left(\|b(y)\|_{L^{\infty}}\right), \quad C:=\|f\|_{H^{-1}} .
$$

This motivates the study of the finiteness of exponential moments of $\|b(y)\|_{L^{\infty}}$ which we formulate for any given $0 \leq k<\infty$ as follows.

Property $\mathbf{M}_{k}$ : The map $y \mapsto b(y)$ is measurable from $U$ to $L^{\infty}(D)$, and the exponential moment $\mathbb{E}\left(\exp \left(k\|b(y)\|_{L^{\infty}}\right)\right)$ is finite.

According to (2.4), we find that Property $\mathbf{M}_{k}$ implies the finiteness of the $k$-th moment $\mathbb{E}\left(\|u(y)\|_{V}^{k}\right)$. Several types of conditions on $b$ have been introduced in the literature $[5,10,19]$ which are sufficient to guarantee that Property $\mathbf{M}_{k}$ holds.

One first approach requires that $b=(b(x))_{x \in D}$ is a Gaussian random field with some minimal smoothness, in the sense of a smoothness assumptions on the covariance kernel $C_{b}$. Indeed, if $C_{b}$ belongs to the Hölder space $C^{\beta}(D \times D)$ for some $\beta>0$, that is, there exists $C>0$ such that

$$
\left|C_{b}\left(x, x^{\prime}\right)-C_{b}\left(z, z^{\prime}\right)\right| \leq C\left(|x-z|^{\beta}+\left|x^{\prime}-z^{\prime}\right|^{\beta}\right), \quad x, z, x^{\prime}, z^{\prime} \in D,
$$

then, following the argument of Proposition 2.1 in [5], we first find by using the Kolmogorov continuity theorem that there exists a version of $b$ with trajectories almost surely in $C^{\alpha}(D)$ for $\alpha<\beta / 2$. By application of Fernique's theorem, see Proposition 2.3 of [5], we then find that

$$
\mathbb{E}\left(\exp \left(\lambda\|b\|_{L^{\infty}}^{2}\right)\right) \leq \mathbb{E}\left(\exp \left(\lambda\|b\|_{C^{\alpha}}^{2}\right)\right)<\infty,
$$

for some sufficiently small $\lambda>0$. This implies that $\mathbb{E}\left(\exp \left(k\|b\|_{L^{\infty}}\right)\right)$ is finite for all $0 \leq k<\infty$. Note that when $b$ has the representation (1.10), its covariance kernel is then given by

$$
C_{b}\left(x, x^{\prime}\right)=\sum_{j \geq 1} \psi_{j}(x) \psi_{j}\left(x^{\prime}\right)
$$

and therefore we may analyze the smoothness of $C_{b}$ through that of the individual $\psi_{j}$. A strategy to establish that $b$ with representation (1.10) is almost surely in a Hölder space $C^{\kappa}$ for some $\kappa>0$ is discussed in $[10,18]$. It assumes that the $\psi_{j}$ are individually in $C^{\alpha}$ for some $\alpha>0$, and that for some $0<\delta<2$,

$$
\sum_{j \geq 1}\left\|\psi_{j}\right\|_{L^{\infty}}^{2}<\infty \text { and } \sum_{j \geq 1}\left\|\psi_{j}\right\|_{L^{\infty}}^{2-\delta} C_{j}^{\delta}<\infty
$$

where $C_{j}$ is a constant such that $\left|\psi_{j}(x)-\psi_{j}\left(x^{\prime}\right)\right| \leq C_{j}\left|x-x^{\prime}\right|^{\alpha}$. Then, using the Kolmogorov continuity theorem, one establishes that $b$ belongs almost surely to $C^{\kappa}$ for $\kappa<\alpha \delta / 2$, see Corollary 7.22 in [10] (one can also easily check that (2.8) implies (2.5) with $\beta=\delta$ ).

A second approach for guaranteeing Property $\mathbf{M}_{k}$ does not assume any smoothness on the $\psi_{j}$, but instead the summability property

$$
\sum_{j \geq 1}\left\|\psi_{j}\right\|_{L^{\infty}}<\infty
$$


Then, as observed in [19], we find that for all $0 \leq k<\infty$,

$$
\mathbb{E}\left(\exp \left(k\|b(y)\|_{L^{\infty}}\right)\right) \leq \mathbb{E}\left(\exp \left(k \sum_{j \geq 1}\left|y_{j}\right|\left\|\psi_{j}\right\|_{L^{\infty}}\right)\right)=\prod_{j \geq 1} \mathbb{E}\left(\exp \left(k\left|y_{j}\right|\left\|\psi_{j}\right\|_{L^{\infty}}\right)\right)<\infty,
$$

since for a standard Gaussian variable $t$ and a positive quantity $s$ one has

$$
\mathbb{E}(\exp (s|t|))=\frac{2}{\sqrt{2 \pi}} \int_{0}^{+\infty} \exp \left(s t-\frac{t^{2}}{2}\right) \mathrm{d} t=\mathrm{e}^{\frac{s^{2}}{2}} \frac{2}{\sqrt{2 \pi}} \int_{-s}^{+\infty} \mathrm{e}^{-\frac{t^{2}}{2}} \mathrm{~d} t \leq \exp \left(\frac{s^{2}}{2}+\frac{2}{\sqrt{2 \pi}} s\right) .
$$

Neither of these two types of conditions imply each other. Indeed, on the one hand, if $\left(\psi_{j}\right)_{j \geq 0}$ is a smooth wavelet basis of $L^{2}(D)$ ordered from coarser to finer scale, $C^{\beta}$ smoothness of $C_{b}$ holds whenever $\left\|\psi_{j}\right\|_{L^{\infty}} \leq$ $C j^{-\frac{\beta}{2 d}}$, which is not sufficient to ensure that $\left(\left\|\psi_{j}\right\|_{L^{\infty}}\right)_{j \geq 1}$ belongs to $\ell^{1}(\mathbb{N})$ if $\frac{\beta}{2 d} \leq 1$. This shows that $(2.5)$ may hold while (2.9) may fail. Also note that, for the same reason, the criterion (2.8) is sufficient but not necessary for (2.5) to hold. On the other hand, if the $\psi_{j}$ are discontinuous, we cannot hope that $C_{b}$ has Hölder smoothness while $\left(\left\|\psi_{j}\right\|_{L^{\infty}}\right)_{j \geq 1}$ could belong to $\ell^{1}(\mathbb{N})$, which shows that (2.9) may hold while (2.5) and (2.8) may fail. Note that the exclusion of discontinuous $\psi_{j}$ is problematic when one needs to model sharp interfaces in the diffusion media.

We also remark that conditions (2.8) and (2.9) are artificially strong when the supports of the $\psi_{j}$ do not overlap, or only partially overlap. This motivates us to introduce a third condition which better takes into account the support properties of the $\psi_{j}$, and does not enforce them to be Hölder continuous.

Assumption A: There exists a strictly positive sequence $\rho=\left(\rho_{j}\right)_{j \geq 1}$ such that the series $\sum_{j \geq 1} \rho_{j}\left|\psi_{j}\right|$ converges in $L^{\infty}(D)$ and

$$
\sum_{j \geq 1} \exp \left(-\rho_{j}^{2}\right)<\infty
$$

Note that since the $\rho_{j}$ are strictly positive, the summability of the $\exp \left(-\rho_{j}^{2}\right)$ also implies that

$$
\rho_{0}:=\inf _{j \geq 1} \rho_{j}>0 .
$$

We shall make use of the following elementary lemma.

Lemma 2.1. If $\left(\alpha_{j}\right)_{j \geq 1}$ is a sequence of numbers from $\left[0,1\left[\right.\right.$ that belongs to $\ell^{1}(\mathbb{N})$, then, there is a constant $c>0$ and $M_{0} \geq 1$ such that

$$
\sum_{j=1}^{\infty} \alpha_{j}^{M} \leq \mathrm{e}^{-c M}, \quad M \geq M_{0} .
$$

Proof. We first consider the case when $\sum_{j=1}^{\infty} \alpha_{j}<1$. In this case, (2.14) follows with $M_{0}=1$ because $\left\|\left(\alpha_{j}\right)_{j \geq 1}\right\|_{\ell^{M}} \leq\left\|\left(\alpha_{j}\right)_{j \geq 1}\right\|_{\ell^{1}}$, for any $M \geq 1$. Hence, with $c=-\ln \left\|\left(\alpha_{j}\right)_{j \geq 1}\right\|_{\ell^{1}}$, we arrive at $(2.14)$. To prove the general case, we first choose $K$ so that $\sum_{j>K} \alpha_{j} \leq 1 / 2$. For any $M_{0}>1$ we may write

$$
\sum_{j=1}^{\infty} \alpha_{j}^{M_{0}}=\sum_{j=1}^{K} \alpha_{j}^{M_{0}}+\sum_{j>K} \alpha_{j}^{M_{0}} \leq \sum_{j=1}^{K} \alpha_{j}^{M_{0}}+\frac{1}{2} \leq K\left(\max _{j=1, \ldots, K} \alpha_{j}\right)^{M_{0}}+\frac{1}{2},
$$

and therefore we have

$$
\sum_{j=1}^{\infty} \alpha_{j}^{M_{0}} \leq \frac{3}{4}
$$


for $M_{0}$ sufficiently large. Since $\alpha_{j}^{M}=\left(\alpha_{j}^{M_{0}}\right)^{M / M_{0}}$ we know from the comparison of $\ell^{p}$ norms that

$$
\sum_{j=1}^{\infty} \alpha_{j}^{M} \leq(3 / 4)^{M / M_{0}}, \quad M \geq M_{0}
$$

and the result follows.

We now prove that Assumption A guarantees the measurability and finiteness of the exponential moments of the map $y \mapsto b(y)$. For $J \geq 1$ we introduce the truncation

$$
b_{J}(y)=\sum_{j=1}^{J} y_{j} \psi_{j}
$$

Theorem 2.2. Assumption $\mathbf{A}$ implies that Property $\mathbf{M}_{k}$ holds for any $0 \leq k<\infty$.

Proof. Let us consider a sequence $\left(\rho_{j}\right)_{j \geq 1}$ such that (2.12) holds. For any $t \geq 0$, the complement $\mathcal{E}_{t}^{c}$ of the event

$$
\mathcal{E}_{t}:=\left\{y: \sup _{j \geq 1} \rho_{j}^{-1}\left|y_{j}\right| \leq t\right\}
$$

has measure

$$
\gamma\left(\mathcal{E}_{t}^{c}\right) \leq \sum_{j=1}^{\infty} \gamma\left\{y:\left|y_{j}\right|>t \rho_{j}\right\} \leq \frac{2}{\rho_{0} \sqrt{2 \pi}} \sum_{j=1}^{\infty} \exp \left(-\frac{t^{2} \rho_{j}^{2}}{2}\right),
$$

where we have used the univariate Gaussian tail bound

$$
\int_{|s|>B} g(s) \mathrm{d} s \leq \frac{2}{B \sqrt{2 \pi}} \mathrm{e}^{-\frac{B^{2}}{2}}, \quad B>0 .
$$

Therefore, application of Lemma 2.1 with $\alpha_{j}=\exp \left(-\rho_{j}^{2}\right)$ shows that

$$
\gamma\left(\mathcal{E}_{t}^{c}\right) \leq C \mathrm{e}^{-c t^{2}}, \quad t \geq t_{0},
$$

for certain constants $C, c, t_{0}$. In particular the event

$$
\mathcal{E}:=\cup_{t \geq 0} \mathcal{E}_{t}=\left\{y: \sup _{j \geq 1} \rho_{j}^{-1}\left|y_{j}\right|<\infty\right\},
$$

has full measure, i.e.

$$
\gamma(\mathcal{E})=1
$$

We next observe that the mapping $y \mapsto b_{J}(y)$, with $b_{J}$ defined by $(2.18)$, is measurable from $U$ to $L^{\infty}(D)$ since it is a continuous $L^{\infty}$-valued function of the variables $\left(y_{1}, \ldots, y_{J}\right)$. We observe that if Assumption $\mathbf{A}$ holds, then, for any $y \in \mathcal{E}$, we may define $b(y)$ as the limit in $L^{\infty}(D)$ of $b_{J}(y)$ since

$$
\left\|b_{J}(y)-b(y)\right\|_{L^{\infty}} \leq\left\|\sum_{j>J} \rho_{j}\left|\psi_{j}\right|\right\|_{L^{\infty}} \sup _{j \geq 1} \rho_{j}^{-1}\left|y_{j}\right| \rightarrow 0 \quad \text { as } J \rightarrow+\infty .
$$

Thus, by (2.24), $b_{J}(y)$ converges to $b(y)$ in $L^{\infty}(D)$ for almost every $y \in U$. Therefore $y \mapsto b(y)$ is also a measurable mapping, that is, $b(y)$ is a random variable with values in $L^{\infty}(D)$. 
In addition, for any $s \geq 0$ and $y \in \mathcal{E}_{s}$, we may write

$$
\|b(y)\|_{L^{\infty}} \leq C_{A} s, \quad C_{A}:=\left\|\sum_{j \geq 1} \rho_{j}\left|\psi_{j}\right|\right\|_{L^{\infty}} .
$$

Hence, for $t \geq 1$, we have

$$
P(t):=\gamma\left\{y:\|b(y)\|_{L^{\infty}}>t\right\} \leq \gamma\left(\mathcal{E}_{t / C_{A}}^{c}\right) \leq \frac{2 C_{A}}{\rho_{0} \sqrt{2 \pi}} \sum_{j=1}^{\infty} \exp \left(-\frac{t^{2} \rho_{j}^{2}}{2 C_{A}^{2}}\right),
$$

where we have used (2.20). From Assumption A, we know that the last sum in (2.27) is finite when $t=t_{0}:=$ $\sqrt{2} C_{A}$. Hence, applying Lemma 2.1 with $\alpha_{j}=\exp \left(-\frac{t_{0}^{2} \rho_{j}^{2}}{2 C_{A}^{2}}\right)$, and using the fact that $P(t) \leq 1$ for all $t \geq 0$, we find that

$$
P(t) \leq C \mathrm{e}^{-c t^{2}}
$$

for suitable $c, C>0$ and for all $t \geq 0$. Therefore

$$
\begin{aligned}
\mathbb{E}\left(\exp \left(k\|b(y)\|_{L^{\infty}}\right)\right) & =1+\int_{1}^{+\infty} \gamma\left\{y: \exp \left(k\|b(y)\|_{L^{\infty}}\right)>s\right\} \mathrm{d} s \\
& =1+\int_{0}^{+\infty} k \exp (k t) P(t) \mathrm{d} t<\infty
\end{aligned}
$$

where we have used the change of variable $s=\exp (k t)$ for all $0<k<\infty$. The above also trivially holds when $k=0$.

If we next define $u_{J}(y)$ as the weak solution of (1.5) with $a=a_{J}(y)=\exp \left(b_{J}(y)\right)$, where $b_{J}$ is defined by (2.18), we find that the mapping $y \mapsto u_{J}(y)$ is measurable from $U$ to $V$ since it is a continuous $V$-valued function of the variables $\left(y_{1}, \ldots, y_{J}\right)$. This continuity stems from the following classical stability estimate: two weak solutions $u$ and $\tilde{u}$ of (1.5) with diffusion coefficients $a$ and $\tilde{a}$, respectively, satisfy

$$
\|u-\tilde{u}\|_{V} \leq C\|a-\tilde{a}\|_{L^{\infty}}, \quad C:=\frac{\|f\|_{V *}}{\min \left\{a_{\min }, \tilde{a}_{\min }\right\}^{2}} .
$$

This estimate also shows that the convergence of $b_{J}(y)$ towards $b(y)$ in $L^{\infty}(D)$ for each $y \in \mathcal{E}$ implies the convergence of $u_{J}(y)$ towards $u(y)$ in $V$ for each $y \in \mathcal{E}$, which shows that the mapping $y \mapsto u(y)$ is measurable as an almost everywhere limit of measurable mappings, that is, $u(y)$ is a random variable with values in $V$. By application of (2.4), we thus obtain the following result.

Corollary 2.3. Assumption A implies that $y \mapsto u(y)$ is measurable with values in $V=H_{0}^{1}(D)$ and that $\mathbb{E}\left(\|u(y)\|_{V}^{k}\right)$ is finite for any $0 \leq k<\infty$.

Remark 2.4. Assumption $\mathbf{A}$ is almost necessary for Property $\mathbf{M}_{k}$ to hold in the case where the supports of $\psi_{j}$ do not overlap, in the sense that Property $\mathbf{M}_{k}$ then implies that $\sum_{j \geq 1} \rho_{j}\left|\psi_{j}\right|$ is uniformly bounded. In this case, the uniform boundedness of $\sum_{j \geq 1} \rho_{j}\left|\psi_{j}\right|$ for a sequence $\left(\rho_{j}\right)_{j \geq 1}$ which satisfies (2.12) can be equivalently expressed as follows: there exists $C_{B}>0$ such that with $b_{j}:=\left\|\psi_{j}\right\|_{L^{\infty}}$, one has

$$
\sum_{j \geq 1} \exp \left(-\frac{C_{B}}{b_{j}^{2}}\right)<\infty
$$


Indeed, since $C_{A}:=\left\|\sum_{j \geq 1} \rho_{j}\left|\psi_{j}\right|\right\|_{L^{\infty}}=\sup _{j \geq 1} \rho_{j} b_{j}$, we have (2.30) with $C_{B}=C_{A}^{-2}$. We then have

$$
\gamma\left\{y:\|b(y)\|_{L^{\infty}(D)} \leq t\right\}=\prod_{j \geq 1} \gamma\left\{y:\left|y_{j}\right| \leq \frac{t}{b_{j}}\right\} \leq \prod_{j \geq 1}\left(1-\frac{2 t}{b_{j} \sqrt{2 \pi}} \exp \left(-2 \frac{t^{2}}{b_{j}^{2}}\right)\right),
$$

for any $t>0$, where we have used the lower bound

$$
\int_{|s|>B} g(s) \mathrm{d} s \geq \int_{B<|s|<2 B} g(s) \mathrm{d} s \geq \frac{2 B}{\sqrt{2 \pi}} \exp \left(-2 B^{2}\right) .
$$

It follows that if (2.30) does not hold for any $C_{B}>0$, then $\gamma\left\{y:\|b(y)\|_{L^{\infty}(D)} \leq t\right\}=0$ for all $t>0$, which means that $\|b(y)\|_{L^{\infty}(D)}=\infty$ with probability 1 and therefore Property $\mathbf{M}_{k}$ does not hold.

Remark 2.5. Assumption A always follows from the assumptions of Theorem 1.2. Namely, if the assumptions of Theorem 1.2 are satisfied for some value of $p<2$ and some sequence $\left(\rho_{j}\right)_{j \geq 1}$, then we claim that Assumption $\mathbf{A}$ is satisfied for the sequence $\omega_{j}:=\rho_{j}^{1 / 2}$. Indeed, we know that $\left(\rho_{j}^{-1}\right)_{j \geq 1} \in \ell^{q}(\mathbb{N})$ for $q=\frac{2 p}{2-p}$ and therefore, up to a nondecreasing rearrangement, we have $\omega_{j} \geq C j^{1 / 2 q}, j \geq 1$, for some $C>0$. So, (2.12) of Assumption A follows. The convergence in $L^{\infty}$ follows from

$$
\left\|\sum_{j>J} \omega_{j}\left|\psi_{j}\right|\right\|_{L^{\infty}} \leq\left(\sup _{j>J} \rho_{j}^{-1 / 2}\right)\left\|\sum_{j \geq 1} \rho_{j}\left|\psi_{j}\right|\right\|_{L^{\infty}} \rightarrow 0, \quad \text { as } \quad J \rightarrow+\infty .
$$

\section{HERMite EXPANSIONS AND PARTIAL DERIVATIVES}

We are interested in the summability properties of the multivariate Hermite expansion (1.16). By Parseval's identity, the $L^{2}$ integrability of $u(y)$ with respect to the Gaussian measure $\gamma$ implies the $\ell^{2}$ summability property

$$
\sum_{\nu \in \mathcal{F}}\left\|u_{\nu}\right\|_{V}^{2}<\infty
$$

In order to obtain polynomial approximation results in the mean-square sense, or equivalently, in $L^{2}\left(\mathbb{R}^{\mathbb{N}}, V, \gamma\right)$, we need to establish the $\ell^{p}$ summability

$$
\sum_{\nu \in \mathcal{F}}\left\|u_{\nu}\right\|_{V}^{p}<\infty
$$

for values of $p<2$. For this purpose, we establish $L^{2}$-integrability properties of the partial derivatives of $u$. For any $V$-valued function $y \mapsto w(y)$ defined on $\mathbb{R}^{\mathbb{N}}$, and $j \in \mathbb{N}$, the derivative $\partial_{y_{j}} w$ is defined as the limit, as $h \rightarrow 0$, of the difference quotient

$$
\frac{w\left(y+h e_{j}\right)-w(y)}{h}
$$

provided this limit exists in $V$, where

$$
e_{j}:=\left(\delta_{i, j}\right)_{i \geq 1}
$$

is the Kronecker sequence of index $j$. Higher derivatives $\partial^{\nu} w$ are defined inductively. For $\nu \in \mathcal{F}$, we use the notation

$$
\partial^{\nu} w=\left(\prod_{j \geq 1} \partial_{y_{j}}^{\nu_{j}}\right) w
$$

When $\nu_{j}=0$, the operator $\partial_{y_{j}}^{\nu_{j}}$ is the identity operator and so the above product has only a finite number of factors which are not the identity. 
We also use the standard notation $\operatorname{supp}(\nu):=\left\{j: \nu_{j} \neq 0\right\}$, as well as

$$
|\nu|:=\|\nu\|_{\ell^{1}}=\sum_{j \geq 1} \nu_{j}, \quad \nu \in \mathcal{F}
$$

and

$$
\nu !:=\prod_{j \geq 1} \nu_{j} !, \quad \nu \in \mathcal{F}
$$

with the convention that $0 !=1$. Furthermore, we use the combinatorial notation,

$$
\left(\begin{array}{c}
\nu \\
\mu
\end{array}\right):=\prod_{j \geq 1}\left(\begin{array}{c}
\nu_{j} \\
\mu_{j}
\end{array}\right), \quad \mu, \nu \in \mathcal{F},
$$

with the convention that

$$
\left(\begin{array}{c}
n \\
m
\end{array}\right):=0, \quad \text { if } \quad m>n
$$

In analogy with $y=\left(y_{j}\right)_{j \geq 1}$, we set $\psi=\left(\psi_{j}\right)_{j \geq 1}$ and use the notation

$$
\psi^{\nu}:=\prod_{j \geq 1} \psi_{j}^{\nu_{j}}, \quad \nu \in \mathcal{F}
$$

Finally, with $\leq$ denoting the componentwise partial order between multi-indices, we define

$$
S_{\mu}:=\{\nu \in \mathcal{F}: \nu \leq \mu \text { and } \nu \neq \mu\}, \quad \mu \in \mathcal{F},
$$

which is always a finite set. The following lemma proves that the derivatives $\partial^{\nu} u(y)$ are elements of $V$ and gives a recursive way of computing them. While it is implicitly contained in the proof of Theorem 3.1 in [19], we give it here in an explicit form for completeness.

Lemma 3.1. For any $y$ such that $\|b(y)\|_{L^{\infty}}<\infty$, and any nonzero $\nu \in \mathcal{F}$, the partial derivative $\partial^{\nu} u(y)$ exists and is the solution of the variational problem

$$
\int_{D} a(y) \nabla \partial^{\mu} u(y) \cdot \nabla v \mathrm{~d} x=-\sum_{\nu \in S_{\mu}}\left(\begin{array}{l}
\mu \\
\nu
\end{array}\right) \int_{D} \psi^{\mu-\nu} a(y) \nabla \partial^{\nu} u(y) \cdot \nabla v \mathrm{~d} x, \quad v \in V .
$$

Proof. The proof of this lemma is, in principle, the same as the proof of Theorem 4.2 in [9]. The only difference is that in the theorem of [9] the assumption is that $a$ is affine in $y$, whereas now we assume $a=\mathrm{e}^{b}$ with $b$ affine in $y$. In going further, we indicate only the necessary changes caused by the different diffusion coefficient.

One begins by establishing the validity of the theorem when $\mu=e_{j}$, for any $j \in \mathbb{N}$, by considering the functions $\rho_{h}(y):=\frac{u\left(y+h e_{j}\right)-u(y)}{h}$, for $|h| \leq 1$. Following the reasoning in [9], one derives that $\rho_{h}$ satisfies

$$
\int_{D} a(y) \nabla \rho_{h}(y) \cdot \nabla v \mathrm{~d} x=L_{h}(v), \quad \text { for all } v \in V,
$$

where $L_{h}$ is the linear functional on $V$ given by

$$
L_{h}(v):=-\int_{D} \frac{\mathrm{e}^{h \psi_{j}}-1}{h} a(y) \nabla u\left(y+h e_{j}\right) \cdot \nabla v \mathrm{~d} x .
$$

Using (2.29), one next proves that $L_{h} \rightarrow L$ in $V^{\prime}$ where $L$ is the linear functional

$$
L(v):=-\int_{D} a(y) \psi_{j} \nabla u(y) \cdot \nabla v \mathrm{~d} x
$$

Therefore, taking a limit as $h \rightarrow 0$ in (3.13) proves the theorem for the case $\mu=e_{j}$. The case of any general $\mu \in \mathcal{F}$ now follows by a recursive application of this result. 
The recursive formula (3.12) will be crucial for obtaining sharp estimates for the partial derivatives $\partial^{\mu} u(y)$. For the time being, we use it to establish a crude bound. For each $y$ such that $\|b(y)\|_{L^{\infty}}<\infty$, we introduce a $y$-dependent equivalent norm on $V$,

$$
\|v\|_{a(y)}^{2}:=\int_{D} a(y)|\nabla v|^{2} \mathrm{~d} x, \quad v \in V .
$$

For such $y$, we have the norm equivalence

$$
\exp \left(-\|b(y)\|_{L^{\infty}}\right)\|v\|_{V}^{2} \leq\|v\|_{a(y)}^{2} \leq \exp \left(\|b(y)\|_{L^{\infty}}\right)\|v\|_{V}^{2}, \quad v \in V .
$$

Lemma 3.2. For each y such that $\|b(y)\|_{L^{\infty}}<\infty$, we have

$$
\left\|\partial^{\mu} u(y)\right\|_{V} \leq C \exp \left((2|\mu|+1)\|b(y)\|_{L^{\infty}}\right), \quad \mu \in \mathcal{F},
$$

where the constant depends on $\mu$, on the $\psi_{j}$, and on $f$.

Proof. Using (3.17) in conjunction with (3.12) and $v=\partial^{\mu} u(y)$, we obtain

$$
\begin{aligned}
\left\|\partial^{\mu} u(y)\right\|_{V}^{2} & \leq \mathrm{e}^{\|b(y)\|_{L^{\infty}}}\left\|\partial^{\mu} u(y)\right\|_{a(y)}^{2} \\
& \leq \mathrm{e}^{\|b(y)\|_{L^{\infty}}} \sum_{\nu \in S_{\mu}}\left(\begin{array}{l}
\mu \\
\nu
\end{array}\right)\left(\prod_{j \in \operatorname{supp}(\mu-\nu)}\left\|\psi_{j}\right\|_{L^{\infty}}^{\mu_{j}-\nu_{j}}\right)\left\|\partial^{\nu} u(y)\right\|_{a(y)}\left\|\partial^{\mu} u(y)\right\|_{a(y)} \\
& \leq C_{\mu} \mathrm{e}^{2\|b(y)\|_{L^{\infty}}}\left\|\partial^{\mu} u(y)\right\|_{V} \sum_{\nu \in S_{\mu}}\left\|\partial^{\nu} u(y)\right\|_{V},
\end{aligned}
$$

with a constant $C_{\mu}>0$. Applying this recursively, starting from the estimate (2.4) for $\|u(y)\|_{V}$, we obtain (3.18)

In view of Property $\mathbf{M}_{k}$, the above result guarantees the finiteness of all moments $\mathbb{E}\left(\left\|\partial^{\mu} u(y)\right\|_{V}^{k}\right)$ for all $0 \leq k<\infty$. The following result relates the $L^{2}$ norms of the mixed derivatives of $u$ up to some given order $r$ with weighted $\ell^{2}$ norms of the Hermite coefficients. In this result $r \geq 0$ is an arbitrary integer which will be fixed later.

Theorem 3.3. Let $r \geq 0$ be an integer and let $\rho=\left(\rho_{j}\right)_{j \geq 1}$ be a sequence of positive numbers. Assume that Property $\mathbf{M}_{k}$ hold for all $0 \leq k<\infty$. Then

$$
\sum_{\nu \in \mathcal{F}} b_{\nu}\left\|u_{\nu}\right\|_{V}^{2}=\sum_{\|\mu\|_{\ell \infty} \leq r} \frac{\rho^{2 \mu}}{\mu !} \int_{U}\left\|\partial^{\mu} u(y)\right\|_{V}^{2} \mathrm{~d} \gamma(y),
$$

where

$$
b_{\nu}:=\sum_{\|\mu\|_{\ell \infty} \leq r}\left(\begin{array}{l}
\nu \\
\mu
\end{array}\right) \rho^{2 \mu} .
$$

The weights $b_{\nu}$ can also be expressed as

$$
b_{\nu}=\prod_{j \geq 1}\left(\sum_{l=0}^{r}\left(\begin{array}{c}
\nu_{j} \\
l
\end{array}\right) \rho_{j}^{2 l}\right) .
$$


Proof. With the normalization in $L^{2}(\mathbb{R}, d g)$ that we have chosen for the univariate Hermite polynomials, we have the Rodrigues formula

$$
H_{n}(t)=\frac{(-1)^{n}}{\sqrt{n !}} \frac{g^{(n)}(t)}{g(t)}
$$

As a consequence, for $m \leq n$ and sufficiently smooth univariate functions $v$ with square-integrable derivatives, integration by parts yields

$$
\begin{aligned}
v_{n} & :=\int_{\mathbb{R}} v H_{n} g \mathrm{~d} t=\frac{(-1)^{n}}{\sqrt{n !}} \int_{\mathbb{R}} v g^{(n)} \mathrm{d} t \\
& =\frac{(-1)^{n-m}}{\sqrt{n !}} \int_{\mathbb{R}} v^{(m)} g^{(n-m)} \mathrm{d} t=\sqrt{\frac{(n-m) !}{n !}} \int_{\mathbb{R}} v^{(m)} H_{n-m} g \mathrm{~d} t .
\end{aligned}
$$

By Parseval's identity, we thus have

$$
\frac{1}{m !} \int_{\mathbb{R}}\left|v^{(m)}\right|^{2} g \mathrm{~d} t=\sum_{n \geq m} \frac{n !}{m !(n-m) !}\left|v_{n}\right|^{2}=\sum_{n \geq 0}\left(\begin{array}{c}
n \\
m
\end{array}\right)\left|v_{n}\right|^{2},
$$

where in the last step we have made use of our notational convention (3.9).

For $\nu, \mu \in \mathcal{F}$ with $\mu \leq \nu$, applying the same reasoning to the tensor product Hermite coefficients $u_{\nu}$, we obtain

$$
u_{\nu}=\left\langle u, H_{\nu}\right\rangle=\sqrt{\frac{(\nu-\mu) !}{\nu !}}\left\langle\partial^{\mu} u, H_{\nu-\mu}\right\rangle
$$

Concerning the integration by parts, note that, as a consequence of (3.18), Property $\mathbf{M}_{k}$ allows us to proceed as in (3.23). By Parseval's identity, summation over $\nu \in \mathcal{F}$ with $\nu \geq \mu$ thus gives

$$
\frac{1}{\mu !} \int_{U}\left\|\partial^{\mu} u(y)\right\|_{V}^{2} \mathrm{~d} \gamma(y)=\sum_{\nu \geq \mu} \frac{\nu !}{\mu !(\nu-\mu) !}\left\|u_{\nu}\right\|_{V}^{2}=\sum_{\nu \in \mathcal{F}}\left(\begin{array}{c}
\nu \\
\mu
\end{array}\right)\left\|u_{\nu}\right\|_{V}^{2}
$$

where we have again used (3.9). Multiplying this identity by $\rho^{2 \mu}$ and summing over $\mu$ with $\|\mu\|_{\ell^{\infty}} \leq r$ gives

$$
\sum_{\|\mu\|_{\ell \infty} \leq r} \frac{\rho^{2 \mu}}{\mu !} \int_{U}\left\|\partial^{\mu} u(y)\right\|_{V}^{2} \mathrm{~d} \gamma(y)=\sum_{\|\mu\|_{\ell \infty} \leq r} \sum_{\nu \in \mathcal{F}}\left(\begin{array}{c}
\nu \\
\mu
\end{array}\right) \rho^{2 \mu}\left\|u_{\nu}\right\|_{V}^{2}=\sum_{\nu \in \mathcal{F}} b_{\nu}\left\|u_{\nu}\right\|_{V}^{2}
$$

that is, (3.19) with $b_{\nu}$ as defined in (3.20). The equivalent expression of $b_{\nu}$, given in (3.21), immediately follows by factorization in (3.20).

\section{Estimates of the Partial DeRivatives}

In view of Theorem 3.3, estimating certain weighted $\ell^{2}$ norms of the sequence $\left(\left\|u_{\nu}\right\|_{V}\right)_{\nu \in \mathcal{F}}$ amounts to estimating related weighted $\ell^{2}$ norms of the sequence

$$
\left(\int_{U}\left\|\partial^{\mu} u(y)\right\|_{V}^{2} \mathrm{~d} \gamma(y)\right)_{\|\mu\|_{\ell \infty} \leq r}
$$

which we next address. 


\subsection{Estimates for fixed $y$}

Recalling the norm $\|\cdot\|_{a(y)}$ defined in (3.16), we first fix $y$ and estimate the relevant weighted $\ell^{2}$ norms for the sequence $\left(\left\|\partial^{\mu} u(y)\right\|_{a(y)}\right)_{\|\mu\|_{\ell \infty} \leq r}$.

Theorem 4.1. Let $r \geq 1$ be an integer. Assume that there exists a positive sequence $\left(\rho_{j}\right)_{j \geq 1}$ such that

$$
\sup _{x \in D} \sum_{j \geq 1} \rho_{j}\left|\psi_{j}(x)\right|=: K<C_{r}:=\frac{\ln 2}{\sqrt{r}} .
$$

Then, there exists a constant $C$ that depends on $K$ and $r$, such that

$$
\sum_{\|\mu\|_{\infty} \leq r} \frac{\rho^{2 \mu}}{\mu !}\left\|\partial^{\mu} u(y)\right\|_{a(y)}^{2} \leq C\|u(y)\|_{a(y)}^{2},
$$

for all y satisfying $\|b(y)\|_{L^{\infty}}<\infty$.

Proof. For $k \geq 0$, we define $\Lambda_{k}:=\left\{\mu \in \mathcal{F}:|\mu|=k,\|\mu\|_{\ell^{\infty}} \leq r\right\}$ and

$$
\sigma_{k}:=\sum_{\mu \in \Lambda_{k}} \frac{\rho^{2 \mu}}{\mu !}\left\|\partial^{\mu} u(y)\right\|_{a(y)}^{2} .
$$

We prove that

$$
\sigma_{k} \leq \sigma_{0} \delta^{k}
$$

for a fixed $\delta<1$. Since $\sigma_{0}=\|u(y)\|_{a(y)}^{2}$, the theorem follows from this by summing over $k$.

We introduce the notation

$$
\varepsilon(\mu, \nu):=\frac{\sqrt{\mu !}}{\sqrt{\nu !}} \frac{\rho^{\mu-\nu}|\psi|^{\mu-\nu}}{(\mu-\nu) !},
$$

where $|\psi|^{\nu}:=\prod_{j \geq 1}\left|\psi_{j}\right|^{\nu_{j}}$. From (3.12), recalling the notation (3.11), we have

$$
\sigma_{k} \leq \int_{D} \sum_{\mu \in \Lambda_{k}} \sum_{\nu \in S_{\mu}} \varepsilon(\mu, \nu) a(y) \frac{\rho^{\nu}\left|\nabla \partial^{\nu} u(y)\right|}{\sqrt{\nu !}} \frac{\rho^{\mu}\left|\nabla \partial^{\mu} u(y)\right|}{\sqrt{\mu !}} \mathrm{d} x
$$

and thus, by the Cauchy-Schwarz inequality,

$$
\sigma_{k} \leq \int_{D} \sum_{\mu \in \Lambda_{k}}\left(\sum_{\nu \in S_{\mu}} \varepsilon(\mu, \nu) a(y) \frac{\left|\rho^{\nu} \nabla \partial^{\nu} u(y)\right|^{2}}{\nu !}\right)^{1 / 2}\left(\sum_{\nu \in S_{\mu}} \varepsilon(\mu, \nu) a(y) \frac{\left|\rho^{\mu} \nabla \partial^{\mu} u(y)\right|^{2}}{\mu !}\right)^{1 / 2} \mathrm{~d} x .
$$

For $\mu \in \Lambda_{k}$, define $S_{\mu, \ell}:=\left\{\nu \in S_{\mu}:|\mu-\nu|=\ell\right\}$. We have

$$
\sum_{\nu \in S_{\mu}} \varepsilon(\mu, \nu)=\sum_{\ell=1}^{k} \sum_{\nu \in S_{\mu, \ell}} \varepsilon(\mu, \nu) .
$$

Now, from (4.2), we have

$$
\begin{aligned}
\sum_{\nu \in S_{\mu, \ell}} \varepsilon(\mu, \nu) & \leq r^{\ell / 2} \sum_{\nu \in S_{\mu, \ell}} \frac{\rho^{\mu-\nu}|\psi|^{\mu-\nu}}{(\mu-\nu) !} \leq r^{\ell / 2} \sum_{|\tau|=\ell} \frac{\rho^{\tau}|\psi|^{\tau}}{\tau !} \\
& =r^{\ell / 2} \frac{1}{\ell !}\left(\sum_{j=1}^{\infty} \rho_{j}\left|\psi_{j}(x)\right|\right)^{\ell} \leq r^{\ell / 2} \frac{1}{\ell !} K^{\ell}
\end{aligned}
$$


where in the first line we have used the fact that

$$
\frac{\mu !}{\nu !}=\prod_{j \geq 1} \frac{\mu_{j} !}{\nu_{j} !} \leq \prod_{j \geq 1} \mu_{j}^{\mu_{j}-\nu_{j}} \leq r^{|\mu-\nu|} \leq r^{\ell}
$$

since $\|\mu\|_{\ell \infty} \leq r, \nu \leq \mu$ and $|\mu-\nu|=\ell$.

Substituting (4.10) in the right side of (4.9), we find that

$$
\sum_{\nu \in S_{\mu}} \varepsilon(\mu, \nu) \leq \mathrm{e}^{\sqrt{r} K}-1 \leq \mathrm{e}^{\sqrt{r} C_{r}}-1=1 .
$$

Inserting this into (4.8), and applying the Cauchy-Schwarz inequality, we obtain

$$
\begin{aligned}
\sigma_{k} & \leq \int_{D} \sum_{\mu \in \Lambda_{k}}\left(\sum_{\nu \in S_{\mu}} \varepsilon(\mu, \nu) a(y) \frac{\left|\rho^{\nu} \nabla \partial^{\nu} u(y)\right|^{2}}{\nu !}\right)^{1 / 2}\left(a(y) \frac{\left|\rho^{\mu} \nabla \partial^{\mu} u(y)\right|^{2}}{\mu !}\right)^{1 / 2} \mathrm{~d} x \\
& \leq \int_{D}\left(\sum_{\mu \in \Lambda_{k}} \sum_{\nu \in S_{\mu}} \varepsilon(\mu, \nu) a(y) \frac{\left|\rho^{\nu} \nabla \partial^{\nu} u(y)\right|^{2}}{\nu !}\right)^{1 / 2}\left(\sum_{\mu \in \Lambda_{k}} a(y) \frac{\left|\rho^{\mu} \nabla \partial^{\mu} u(y)\right|^{2}}{\mu !}\right)^{1 / 2} \mathrm{~d} x .
\end{aligned}
$$

We treat the first factor in the last integral by interchanging summations in $\mu$ and $\nu$. For this purpose, we introduce, for any $\ell \leq k-1$ and $\nu \in \Lambda_{\ell}$, the set

$$
R_{\nu, k}:=\left\{\mu \in \Lambda_{k}: \nu \in S_{\mu}\right\}
$$

By a similar argument as above for the set $S_{\mu, \ell}$, we find that

$$
\sum_{\mu \in R_{\nu, k}} \varepsilon(\mu, \nu) \leq r^{(k-\ell) / 2} \frac{1}{(k-\ell) !} K^{k-\ell}
$$

It follows that

$$
\sigma_{k} \leq \int_{D}\left(\sum_{\ell=0}^{k-1} r^{(k-\ell) / 2} \frac{1}{(k-\ell) !} K^{k-\ell} \sum_{\nu \in \Lambda_{\ell}} a(y) \frac{\left|\rho^{\nu} \nabla \partial^{\nu} u(y)\right|^{2}}{\nu !}\right)^{1 / 2}\left(\sum_{\mu \in \Lambda_{k}} a(y) \frac{\left|\rho^{\mu} \nabla \partial^{\mu} u(y)\right|^{2}}{\mu !}\right)^{1 / 2} \mathrm{~d} x .
$$

If we now apply the Cauchy-Schwarz inequality to the above integral we obtain

$$
\begin{aligned}
\sigma_{k} & \leq\left(\sum_{\ell=0}^{k-1} r^{(k-\ell) / 2} \frac{1}{(k-\ell) !} K^{k-\ell} \int_{D} \sum_{\nu \in \Lambda_{\ell}} a(y) \frac{\left|\rho^{\nu} \nabla \partial^{\nu} u(y)\right|^{2}}{\nu !} \mathrm{d} x\right)^{1 / 2}\left(\sum_{\mu \in \Lambda_{k}} \int_{D} a(y) \frac{\left|\rho^{\mu} \nabla \partial^{\mu} u(y)\right|^{2}}{\mu !} \mathrm{d} x\right)^{1 / 2} \\
& =\left(\sum_{\ell=0}^{k-1} \frac{1}{(k-\ell) !}(\sqrt{r} K)^{k-\ell} \sigma_{\ell}\right)^{1 / 2} \sigma_{k}^{1 / 2}
\end{aligned}
$$

In other words,

$$
\sigma_{k} \leq \sum_{\ell=0}^{k-1} \frac{1}{(k-\ell) !}(\sqrt{r} K)^{k-\ell} \sigma_{\ell}
$$

Now pick $\delta<1$ such that $K / \delta \leq C_{r}$. By induction, we prove that $\sigma_{k} \leq \sigma_{0} \delta^{k}$ for all $k \geq 0$. This is clearly true for $k=0$ and assuming it has been proven for $k-1$, we find

$$
\sigma_{k} \leq \sigma_{0} \sum_{\ell=0}^{k-1} \frac{1}{(k-\ell) !}(\sqrt{r} K)^{k-\ell} \delta^{\ell} \leq \sigma_{0} \delta^{k} \sum_{\ell=0}^{k-1} \frac{1}{(k-\ell) !}\left(\sqrt{r} C_{r}\right)^{k-\ell} \leq \sigma_{0} \delta^{k}\left(\mathrm{e}^{\sqrt{r} C_{r}}-1\right)=\sigma_{0} \delta^{k},
$$

which advances the induction. This establishes (4.5) and completes the proof of the theorem. 


\subsection{Integral estimates}

Combining (3.17) with Theorem 4.1, we obtain the following result.

Theorem 4.2. Let $r \geq 1$ be an integer. Assume that there exists a positive sequence $\left(\rho_{j}\right)_{j \geq 1}$ such that

$$
\sup _{x \in D} \sum_{j \geq 1} \rho_{j}\left|\psi_{j}(x)\right|=: K<C_{r}:=\frac{\ln 2}{\sqrt{r}} .
$$

Assume in addition that Property $\mathbf{M}_{k}$ holds for $k=4$. Then we have

$$
\sum_{\|\mu\|_{\ell} \leq r} \frac{\rho^{2 \mu}}{\mu !} \int_{U}\left\|\partial^{\mu} u(y)\right\|_{V}^{2} \mathrm{~d} \gamma(y)<\infty .
$$

Proof. We apply (3.17) with $v=\partial^{\mu} u(y)$ and obtain

$$
\left\|\partial^{\mu} u(y)\right\|_{V}^{2} \leq \exp \left(\|b(y)\|_{L^{\infty}}\right)\left\|\partial^{\mu} u(y)\right\|_{a(y)}^{2} .
$$

This gives

$$
\begin{aligned}
\sum_{\|\mu\|_{\ell \infty} \leq r} \frac{\rho^{2 \mu}}{\mu !} \int_{U}\left\|\partial^{\mu} u(y)\right\|_{V}^{2} \mathrm{~d} \gamma(y) & \leq \int_{U} \exp \left(\|b(y)\|_{L^{\infty}}\right) \sum_{\|\mu\|_{\ell \infty} \leq r} \frac{\rho^{2 \mu}}{\mu !}\left\|\partial^{\mu} u(y)\right\|_{a(y)}^{2} \mathrm{~d} \gamma(y) \\
& \leq C_{1} \int_{U} \exp \left(\|b(y)\|_{L^{\infty}}\right)\|u(y)\|_{a(y)}^{2} \mathrm{~d} \gamma(y) \\
& \leq C_{1} \int_{U} \exp \left(2\|b(y)\|_{L^{\infty}}\right)\|u(y)\|_{V}^{2} \mathrm{~d} \gamma(y) \\
& \leq C_{2} \int_{U} \exp \left(4\|b(y)\|_{L^{\infty}}\right) \mathrm{d} \gamma(y)
\end{aligned}
$$

where the second inequality uses Theorem 4.1, the third inequality uses (3.17) again, and the last inequality uses (2.4). From Property $\mathbf{M}_{4}$, the last integral is finite and the proof of the theorem is complete.

\section{Summability of Hermite coefficients}

According to Theorem 3.3, the conclusion of Theorem 4.2 also gives the weighted $\ell^{2}$ summability estimate

$$
\sum_{\nu \in \mathcal{F}} b_{\nu}\left\|u_{\nu}\right\|_{V}^{2}<\infty
$$

Using Hölder's inquality, for $0<p<2$ and $q:=\frac{2 p}{2-p}$ we thus have

$$
\sum_{\nu \in \mathcal{F}}\left\|u_{\nu}\right\|_{V}^{p} \leq\left(\sum_{\nu \in \mathcal{F}} b_{\nu}\left\|u_{\nu}\right\|_{V}^{2}\right)^{p / 2}\left(\sum_{\nu \in \mathcal{F}} b_{\nu}^{-q / 2}\right)^{1-p / 2} .
$$

The following result allows us to control the second factor.

Lemma 5.1. Let $0<p<2$ and $q:=\frac{2 p}{2-p}$ and let $\left(\rho_{j}\right)_{j \geq 1}$ be a positive sequence such that

$$
\left(\rho_{j}^{-1}\right)_{j \geq 1} \in \ell^{q}(\mathbb{N}) .
$$

Then, for any positive integer $r$ such that $\frac{2}{r+1}<p$, the sequence $\left(b_{\nu}\right)$ defined in (3.20) for this $r$ satisfies

$$
\sum_{\nu \in \mathcal{F}} b_{\nu}^{-q / 2}<\infty
$$


Proof. The sum under consideration can be rewritten in factorized form as

$$
\sum_{\nu \in \mathcal{F}} b_{\nu}^{-q / 2}=\sum_{\nu \in \mathcal{F}} \prod_{j \geq 1}\left(\sum_{l=0}^{r}\left(\begin{array}{c}
\nu_{j} \\
l
\end{array}\right) \rho_{j}^{2 l}\right)^{-q / 2}=\prod_{j \geq 1} \sum_{n \geq 0}\left(\sum_{l=0}^{r}\left(\begin{array}{l}
n \\
l
\end{array}\right) \rho_{j}^{2 l}\right)^{-q / 2}
$$

provided we can show that the product on the right side is finite. Now,

$$
\sum_{n \geq 0}\left(\sum_{l=0}^{r}\left(\begin{array}{c}
n \\
l
\end{array}\right) \rho_{j}^{2 l}\right)^{-q / 2} \leq \sum_{n \geq 0}\left[\left(\begin{array}{c}
n \\
n \wedge r
\end{array}\right) \rho_{j}^{2(n \wedge r)}\right]^{-q / 2}=\sum_{n=0}^{r-1} \rho_{j}^{n q}+C_{r, q} \rho_{j}^{-r q},
$$

where in the first inequality we use that the value $l=n \wedge r:=\min \{n, r\}$ is contained in the sum, and where

$$
C_{r, q}:=\sum_{n \geq r}\left(\begin{array}{l}
n \\
r
\end{array}\right)^{-q / 2}=(r !)^{q / 2} \sum_{n \geq 0}[(n+1) \cdots(n+r)]^{-q / 2} .
$$

Since each term in the sum behaves like $n^{-r q / 2}$ as $n \rightarrow \infty$, we find that $C_{r, q}<\infty$ if and only if $q>2 / r$, which holds precisely under our assumption $p>\frac{2}{r+1}$. Since $\rho_{j} \rightarrow \infty$, there exists $J$ such that $\rho_{j}>1$ for all $j>J$. For such $j$, we can bound the right side of (5.6) by $1+\left(C_{r, q}+r-1\right) \rho_{j}^{-q}$. Hence, returning to (5.5), for a finite constant $C$ depending on $w$, we have

$$
\sum_{\nu \in \mathcal{F}} b_{\nu}^{-q / 2} \leq C \prod_{j>J}\left(1+\left(C_{r, q}+r-1\right) \rho_{j}^{-q}\right)
$$

where the product on the right side converges because $\sum_{j \geq 1} \rho_{j}^{-q}<\infty$.

We are now ready to prove our main result.

Proof of Theorem 1.2. By our assumptions, we are given a positive sequence $\left(\rho_{j}\right)_{j \geq 1}$ such that

$$
\sup _{x \in D} \sum_{j \geq 1} \rho_{j}\left|\psi_{j}(x)\right|<\infty \quad \text { and } \quad\left(\rho_{j}^{-1}\right)_{j \geq 1} \in \ell^{q}(\mathbb{N}), \quad q:=\frac{2 p}{2-p} .
$$

We choose $r$ as the minimal integer satisfying $\frac{2}{r+1}<p$. Since the sequence $\rho_{j}$ can be rescaled by multiplying by an arbitrary constant without affecting (5.9), we can assume without loss of generality that

$$
\sup _{x \in D} \sum_{j \geq 1} \rho_{j}\left|\psi_{j}(x)\right|<\frac{\ln 2}{\sqrt{r}} .
$$

Since, as noted in Remark 2.5, $\left(\rho_{j}^{-1}\right)_{j \geq 1} \in \ell^{q}(\mathbb{N})$ implies Assumption A, by Theorem 2.2 we consequently also have Property $\mathbf{M}_{k}$ for all $k$. We can thus combine Theorems 3.3 and 4.2 to obtain

$$
\sum_{\nu \in \mathcal{F}} b_{\nu}\left\|u_{\nu}\right\|_{V}^{2}=\sum_{\|\mu\|_{\ell \infty} \leq r} \frac{\rho^{2 \mu}}{\mu !} \int_{U}\left\|\partial^{\mu} u(y)\right\|_{V}^{2} \mathrm{~d} \gamma(y)<\infty .
$$

Using this together with Lemma 5.1 in (5.2), we arrive at the conclusion.

Remark 5.2. As stated in Theorem 1.2, application of Stechkin's estimate gives that the best $n$-term approximation polynomials

$$
u_{\Lambda_{n}}:=\sum_{\nu \in \Lambda_{n}} u_{\nu} H_{\nu},
$$


obtained by retaining the indices of the $n$ largest $\left\|u_{\nu}\right\|_{V}$, satisfy the estimate

$$
\left\|u-u_{\Lambda_{n}}\right\|_{L^{2}(U, V, \gamma)} \lesssim n^{-s},
$$

where $s:=\frac{1}{p}-\frac{1}{2}=\frac{1}{q}$. There is, however, a more direct and constructive way of retrieving this convergence rate, namely taking instead $\Lambda_{n}$ to be the set of indices corresponding to the $n$ smallest values of the weights $b_{\nu}$ which appear in (5.1). We then directly obtain that

$$
\left\|u-u_{\Lambda_{n}}\right\|_{L^{2}(U, V, \gamma)} \leq \sup _{\nu \notin \Lambda_{n}} b_{\nu}^{-1 / 2}\left(\sum_{\nu \in \mathcal{F}} b_{\nu}\left\|u_{\nu}\right\|_{V}^{2}\right)^{1 / 2} \lesssim d_{n+1}^{*},
$$

where $\left(d_{n}^{*}\right)_{n \geq 1}$ is the decreasing rearrangement of the sequence $\left(b_{\nu}^{-1 / 2}\right)_{\nu \in \mathcal{F}}$. As seen in Lemma 5.1 , this sequence belongs to $\ell^{q}(\mathcal{F})$ which implies that $d_{n}^{*} \lesssim n^{-s}$ with $s:=\frac{1}{q}$. Note that the sets $\Lambda_{n}$ obtained by this strategy depend on the choice of the sequence $\left(\rho_{j}\right)_{j \geq 1}$. All such sets therefore produce the approximation rate $n^{-s}$ (possibly with different multiplicative constants) provided that the chosen sequence satisfies the assumption (5.9).

\section{EXAmples}

In this section, we present several examples of applications of Theorem 1.2 corresponding to different support properties of the $\left(\psi_{j}\right)_{j \geq 1}$. In each case, we discuss which range of $\ell^{q}$ summability of the sequence $\left(\left\|\psi_{j}\right\|_{j \geq 1}\right)_{j \geq 1}$ implies $\ell^{p}$ summability of the sequence $\left(\left\|u_{\nu}\right\|_{V}\right)_{\nu \in \mathcal{F}}$ for some $p<2$.

\subsection{Finitely overlapping supports}

We say that the family $\left(\psi_{j}\right)_{j \geq 1}$ has finitely overlapping supports if and only if there exists an integer $M$ such that for every $x \in D$,

$$
\#\left\{j: \psi_{j}(x) \neq 0\right\} \leq M
$$

One example with $M=1$, which corresponds to disjoint supports, is the set of characteristic functions

$$
\psi_{j}=c_{j} \chi_{D_{j}}
$$

with some normalizing factor $c_{j}$, when $\left(D_{j}\right)_{j \geq 1}$ is a partition of $D$. Another example with $M \geq 1$ is the set of Lagrange finite element basis functions of a given order $k \geq 1$, associated to a conforming simplicial partition of $D$.

For such families, we find that the choice

$$
\rho_{j}^{-1}:=\left\|\psi_{j}\right\|_{L^{\infty}}
$$

yields

$$
\sup _{x \in D} \sum_{j \geq 1} \rho_{j}\left|\psi_{j}(x)\right| \leq M,
$$

and therefore condition (1.26) in Theorem 1.2 is satisfied. We thus obtain the following immediate corollary.

Corollary 6.1. Let $\left(\psi_{j}\right)_{j \geq 1}$ be a family with finitely overlapping supports, and let $0<p<2$. If $\left(\left\|\psi_{j}\right\|_{L^{\infty}}\right)_{j \geq 1}$ belongs to $\ell^{q}(\mathbb{N})$ for $q=q(p):=\frac{2 p}{2-p}$, then $\left(\left\|u_{\nu}\right\|_{V}\right)_{\nu \in \mathcal{F}}$ belongs to $\ell^{p}(\mathcal{F})$. In particular, best $n$-term Hermite approximations converge in $L^{2}(U, V, \gamma)$ with rate $n^{-s}$ where $s=\frac{1}{p}-\frac{1}{2}=\frac{1}{q}$.

As already observed, we always have $q(p)>p$, which shows that there is in this case an improvement in the summability properties of $\left(\left\|u_{\nu}\right\|_{V}\right)_{\nu \in \mathcal{F}}$ over those of $\left(\left\|\psi_{j}\right\|_{L^{\infty}}\right)_{j \geq 1}$. For example, $\ell^{2}$ summability of $\left(\left\|\psi_{j}\right\|_{L^{\infty}}\right)_{j \geq 1}$ implies $\ell^{1}$ summability of $\left(\left\|u_{\nu}\right\|_{V}\right)_{\nu \in \mathcal{F}}$, and therefore convergence of best $n$-term Hermite approximations with rate $n^{-1 / 2}$. 


\subsection{Wavelets}

For a general wavelet bases on a domain $D \subset \mathbb{R}^{d}$, we adopt the notation $\left(\psi_{\lambda}\right)$, used for example in [7], where $\lambda$ concatenates the scale and spatial indices, with the convention that the scale level $l$ of $\psi_{\lambda}$ is denoted by $|\lambda|$, i.e., $|\lambda|:=l$. Thus, there are $\mathcal{O}\left(2^{d l}\right)$ wavelets at level $l$ and the wavelets at each given scale have finite overlap. This means that for all $x \in D$,

$$
\#\left\{\lambda:|\lambda|=l \text { and } \psi_{\lambda}(x) \neq 0\right\} \leq M,
$$

for some fixed $M>0$ independent of $l$. We consider wavelets normalized such that

$$
\left\|\psi_{\lambda}\right\|_{L^{\infty}}=c_{l}=C 2^{-\alpha l}, \quad|\lambda|=l,
$$

for some fixed $C>0$ and $\alpha>0$. Using the finite overlapping property, we find that for any $0<\kappa<\alpha$ the sequence

$$
\rho_{\lambda}:=2^{\kappa|\lambda|},
$$

satisfies

$$
\sup _{x \in D} \sum_{\lambda} \rho_{\lambda}\left|\psi_{\lambda}(x)\right| \leq C M \sum_{l \geq 0} 2^{(\kappa-\alpha) l}<\infty
$$

which is (1.26) in Theorem 1.2.

Note that if we order our wavelet basis from coarse to fine scale, we find for the resulting system $\left(\psi_{j}\right)_{j \geq 1}$ and sequence $\left(\rho_{j}\right)_{j \geq 1}$ the algebraic behaviour

$$
\left\|\psi_{j}\right\|_{L^{\infty}} \sim j^{-\alpha / d}
$$

and

$$
\rho_{j} \sim j^{\kappa / d} .
$$

We thus obtain from Theorem 1.2 the following immediate corollary.

Corollary 6.2. Let $\left(\psi_{j}\right)_{j \geq 1}$ be a wavelet basis with the normalization (6.6). If $\left(\left\|\psi_{j}\right\|_{L^{\infty}}\right)_{j \geq 1}$ belongs to $\ell^{q}(\mathbb{N})$ then $\left(\left\|u_{\nu}\right\|_{V}\right)_{\nu \in \mathcal{F}}$ belongs to $\ell^{p}(\mathcal{F})$ for all $p$ such that $\frac{2 p}{2-p}>q$. In particular, the best $n$-term Hermite approximations converge in $L^{2}(U, V, \gamma)$ with rate $n^{-s}$ for all $s<\frac{1}{q}$.

Proof. Since $\left(\left\|\psi_{j}\right\|_{L^{\infty}}\right)_{j \geq 1}$ belongs to $\ell^{q}(\mathbb{N})$, we know that $q \alpha>d$. Since for the given $p$, we have $q^{*}:=\frac{2 p}{2-p}>q$, we can take $\kappa \in] \frac{d}{q^{*}}, \alpha\left[\right.$ and $\left(\rho_{\lambda}\right)$ as defined in (6.7) for this $\kappa$. Then, the conditions of Theorem 1.2 are satisfied for this sequence of weights and for $q^{*}$. Hence, $\left(\left\|u_{\nu}\right\|_{V}\right)_{\nu \in \mathcal{F}}$ belongs to $\ell^{p}(\mathcal{F})$. This also gives that the approximation rate is $n^{-s}$ with $s=1 / p-1 / 2=1 / q^{*}$. By adjusting $p$, we can take $q^{*}<q$ as close to $q$ as we wish, thereby establishing the rate of convergence $n^{-s}$ for all $s<1 / q$.

Note that if we use sufficiently smooth wavelets, the decay property (6.6) is equivalent to the property that the correlation function $C_{b}$ belongs to the Besov space $B_{\infty}^{\alpha}\left(L^{\infty}(D)\right)$, which coincides with the Hölder space $C^{\alpha}$ when $\alpha$ is non-integer, and therefore $b$ is almost surely in the Hölder space $C^{\beta}$ for $\beta<\alpha / 2$. Thus, we also infer from Theorem 1.2 that if $C_{b}$ belongs to the Besov space $B_{\infty}^{\alpha}\left(L^{\infty}(D)\right)$ for some $\alpha>0$, then best $n$-term Hermite approximations converge in $L^{2}(U, V, \gamma)$ with rate $n^{-s}$ for all $s<\alpha / d$.

\subsection{Arbitrary supports}

We finally consider functions $\psi_{j}$ with arbitrary supports, including the case of globally supported functions such as the Fourier basis. Let us assume that $\left(\left\|\psi_{j}\right\|_{L^{\infty}}\right)_{j \geq 1} \in \ell^{q}(\mathbb{N})$ for some $0<q<1$. We then find that the choice

$$
\rho_{j}:=\left\|\psi_{j}\right\|_{L^{\infty}}^{q-1},
$$

obviously satisfies (1.26). We also find that $\left(\rho_{j}^{-1}\right)_{j \geq 1}$ belongs to $\ell^{r}(\mathbb{N})$ for $r:=\frac{q}{1-q}$. Therefore, applying Theorem 1.2, we find that $\left(\left\|u_{\nu}\right\|_{V}\right)_{\nu \in \mathcal{F}} \in \ell^{p}(\mathcal{F})$ when $p$ satisfies $r=\frac{2 p}{2-p}$ or equivalently $\frac{1}{q}=\frac{1}{p}+\frac{1}{2}$. Therefore, we obtain the following immediate corollary. 
Corollary 6.3. Let $\left(\psi_{j}\right)_{j \geq 1}$ be a family of functions with arbitrary support, and let $0<p<2$. If $\left(\left\|\psi_{j}\right\|_{L^{\infty}}\right)_{j \geq 1}$ belongs to $\ell^{q}(\mathbb{N})$ with $\frac{1}{q}=\frac{1}{p}+\frac{1}{2}$, then $\left(\left\|u_{\nu}\right\|_{V}\right)_{\nu \in \mathcal{F}}$ belongs to $\ell^{p}(\mathcal{F})$. In particular, best $n$-term Hermite approximations converge in $L^{2}(U, V, \gamma)$ with rate $n^{-s}$ for $s=\frac{1}{q}-1$.

Note that if $\left(j^{\beta}\left\|\psi_{j}\right\|_{L^{\infty}}\right)_{j \geq 1} \in \ell^{p}(\mathbb{N})$ for some $\beta>\frac{1}{2}$, an application of Hölder's inequality shows that $\left(\left\|\psi_{j}\right\|_{L^{\infty}}\right)_{j \geq 1}$ belongs to $\ell^{q}(\mathbb{N})$ with $\frac{1}{q}=\frac{1}{p}+\frac{1}{2}$. Therefore, the above corollary represents an improvement over the condition $\left(j\left\|\psi_{j}\right\|_{L^{\infty}}\right)_{j \geq 1} \in \ell^{p}(\mathbb{N})$ from [19].

\section{Non-optimality of the Karhunen-LoÈve Representation}

The previous examples illustrate the role of the support properties of the functions $\left(\psi_{j}\right)_{j \geq 1}$ when analyzing the convergence rate of best $n$-term Hermite approximations for the map $y \mapsto u(y)$. In particular, they reveal that faster convergence rates can be obtained in the case of locally supported functions.

We now discuss a concrete example which illustrates this phenomenon for the approximation of a PDE with given lognormal coefficients, when we use two different representations of these coefficients. Here we take

$$
D=] 0,1[
$$

and therefore consider the equation

$$
-\left(a u^{\prime}\right)^{\prime}=f, \quad u(0)=u(1)=0 .
$$

We take $a=\exp (b)$ where $b$ is a Brownian bridge, that is, a Gaussian process with covariance given by

$$
C_{b}\left(x, x^{\prime}\right)=\min \left\{x, x^{\prime}\right\}-x x^{\prime} .
$$

There exists two simple explicit representations for this process.

(1) The Karhunen-Loève representation is determined by the eigenfunctions $\varphi_{j}$ and eigenvalues $\lambda_{j}$ of the covariance operator, which have the form

$$
\varphi_{j}(x):=\sqrt{2} \sin (\pi j x) \quad \text { and } \quad \lambda_{j}:=\frac{1}{\pi^{2} j^{2}}, \quad j \geq 1,
$$

so that after normalization, we obtain the representation

$$
b(y)=\sum_{j \geq 1} y_{j} \psi_{j}, \quad \psi_{j}(x):=\frac{\sqrt{2}}{\pi j} \sin (\pi j x),
$$

where the $y_{j}$ are i.i.d. standard Gaussian random variables.

(2) The Levy-Ciesielki representation uses the Schauder basis functions, which are the primitives of the Haar functions, namely

$$
\psi_{l, k}(x)=2^{-l / 2} \psi\left(2^{l} x-k\right), \quad k=0, \ldots, 2^{l}-1, l \geq 0,
$$

where $\psi(x):=\max \{0,1 / 2-|x-1 / 2|\}$. Then setting $\psi_{j}=\psi_{l, k}$ when $j=2^{l}+k$, we have again

$$
b(y)=\sum_{j \geq 1} y_{j} \psi_{j},
$$

where the $y_{j}$ are i.i.d. standard Gaussian random variables. 
If we use the Karhunen-Loève representation, the analysis carried in this paper does not allow us to establish any $\ell^{p}$ summablity of $\left(\left\|u_{\nu}\right\|_{V}\right)_{\nu \in \mathcal{F}}$ for $p<2$. Indeed, due to the global nature of the functions $\psi_{j}$ we need to rely on Corollary 6.3 which requires that $\left(\left\|\psi_{j}\right\|_{L^{\infty}}\right)_{j \geq 1} \in \ell^{q}(\mathbb{N})$ for some $q<1$, which does not hold since this sequence is not $\ell^{1}$ summable. Note however that $\ell^{2}$ summability is ensured since $\mathbb{E}\left(\|u(y)\|_{V}^{2}\right)<\infty$.

In contrast, when using the Levy-Ciesielski representation, which is essentially of wavelet type with $\alpha=\frac{1}{2}$ in (6.6), we can rely on Corollary 6.2 which shows that $\left(\left\|u_{\nu}\right\|_{V}\right)_{\nu \in \mathcal{F}}$ belongs to $\ell^{p}(\mathcal{F})$ for all $1<p<2$. In particular, best $n$-term Hermite approximations converge in $L^{2}(U, V, \gamma)$ with rate $n^{-s}$ for all $0<s<1$.

This example reveals that for a given lognormal process, the fastest convergence rates of the best $n$-term Hermite approximation that can be obtained by our currently available analysis, require using a more local representation than Karhunen-Loève. One heuristic explanation of this fact is that the Karhunen-Loève representation is optimal in a very specific sense: it minimizes the mean-square $L^{2}(D)$-error when truncating $b$ by the $J$ first terms in its expansion. However, in the present setting of the elliptic diffusion equation, the relevant norm for approximating the functions $a$ and $b$ is not the $L^{2}$ norm, but rather the $L^{\infty}$ norm for which the Karhunen-Loève representation has no particular optimality property.

\section{REFERENCES}

[1] I. Babuška, F. Nobile and R. Tempone, A stochastic collocation method for elliptic partial differential equations with random input data. SIAM J. Numer. Anal. 45 (2007) 1005-1034.

[2] M. Bachmayr, A. Cohen and G. Migliorati, Sparse polynomial approximation of parametric elliptic PDEs. Part I: Affine coefficients, ESAIM: M2AN $\mathbf{5 1}$ (2017) 321-339.

[3] J. Beck, F. Nobile, L. Tamellini and R. Tempone, On the optimal polynomial approximation of stochastic PDEs by Galerkin and collocation methods. Math. Models Methods Appl. Sci. 22 (2012) 1-33.

[4] J. Beck, F. Nobile, L. Tamellini and R. Tempone, Convergence of quasi-optimal stochastic Galerkin methods for a class of PDEs with random coefficients. Comput. Math. Appl. 67 (2014) 732-751.

[5] J. Charrier, Strong and weak error estimates for elliptic partial differential equations with random coefficients. SIAM J. Numer. Anal. 50 (2012) 216-246.

[6] A. Chkifa, A. Cohen and C. Schwab, Breaking the curse of dimensionality in sparse polynomial approximation of parametric PDEs. J. Math. Pures Appl. 103 (2015) 400-428.

[7] A. Cohen, Numerical analysis of wavelet methods, Studies in Mathematics and its Applications. Elsevier, Amsterdam (2003).

[8] A. Cohen and R. DeVore, Approximation of high-dimensional parametric PDEs. Acta Numer. 24 (2015) 1-159.

[9] A. Cohen, R. DeVore and C. Schwab, Analytic regularity and polynomial approximation of parametric and stochastic PDEs. Anal. Appl. 9 (2011) 11-47.

[10] M. Dashti and A.M. Stuart, The Bayesian Approach to Inverse Problems. Handbook of Uncertainty Quantification, edited by R. Ghanem, D. Higdon and H. Owhadi. Springer (2015).

[11] R. DeVore, Nonlinear Approximation, Acta Numer. 7 (1998) 51-150.

[12] O. Ernst and B. Sprungk, Stochastic Collocation for Elliptic PDEs with Random Data: The Lognormal Case, in Sparse Grids and Applications - Munich (2012), edited by J. Garcke and D. Pflüger. Vol. 97 of Lect. Notes Comput. Sci. Eng. Springer International Publishing Switzerland (2014).

[13] J. Galvis and M. Sarkis, Approximating infinity-dimensional stochastic Darcy's equations without uniform ellipticity. SIAM J. Numer. Anal. 47 (2009) 3624-3651.

[14] R. Ghanem and P. Spanos, Spectral techniques for stochastic finite elements. Arch. Comput. Methods Engrg. 4 (1997) 63-100.

[15] R.G. Ghanem and P.D. Spanos, Stochastic Finite Elements: A Spectral Approach, 2nd edition, Dover (2007).

[16] C. Gittelson, Stochastic Galerkin discretization of the log-normal isotropic diffusion problem. Math. Models Methods Appl. Sci. 20 (2010) 237-263.

[17] I.G. Graham, F.Y. Kuo, J.A. Nichols, R. Scheichl, Ch. Schwab and I. H. Sloan, Quasi-Monte Carlo finite element methods for elliptic PDEs with lognormal random coefficients. Numer. Math. 131 (2015) 329-368.

[18] M. Hairer, An Introduction to Stochastic PDEs. Lecture notes. Available at http://www.hairer.org (2009).

[19] V.H. Hoang and C. Schwab, $N$-term Galerkin Wiener chaos approximation rates for elliptic PDEs with lognormal Gaussian random inputs. M3AS 24 (2014) 797-826.

[20] O. Knio and O. Le Maitre, Spectral Methods for Uncertainty Quantication: With Applications to Computational Fluid Dynamics. Springer (2010).

[21] F.Y. Kuo, R. Scheichl, Ch. Schwab, I.H. Sloan and E. Ullmann, Multilevel Quasi-Monte Carlo Methods for Lognormal Diffusion Problems, arXiv:1507.01090, to appear in Math. of Comp. (2015).

[22] A. Mugler and H.-J. Starkloff, On the convergence of the stochastic Galerkin methods for random elliptic partial differential equations. ESAIM: M2AN $4 \mathbf{7}$ (2013) 1237-1263.

[23] D. Xiu, Numerical Methods for Stochastic Computations: A Spectral Method Approach. Princeton University Press (2010). 